\title{
Convergent Renormalization Expansions for Lattice Gauge Theories
}

\author{
T. Bałaban* \\ Department of Mathematics, Boston University, Boston, MA 02215, USA
}

\begin{abstract}
In this paper we introduce an inductive description of the complete effective densities including large field domains, and we show that the renormalization transformations preserve the form of the densities. This completes the renormalization group analysis for superrenormalizable models and yields convergent expansions in this case.
\end{abstract}

\section{Introduction}

In this paper we continue our study of the renormalization group analysis of the non-Abelian lattice gauge field theories. In the previous papers, referred to as [I, II], we have analyzed the renormalization transformations in the small field regions. The basic goal here is to do such an analysis for the complete model, without any restrictions on field variables, i. e. including all large field regions. Thus we study the complete renormalization transformations $T$, without any restrictions on integration variables. Let us recall that they are of the form

$$
(T \varrho)(V)=\int d U \delta\left(\bar{U} V^{-1}\right) \varrho(U),
$$

where $\varrho(U)$ is a function of the gauge field variables $U$ on a lattice $T, \bar{U}$ is the averaged field on the lattice $T^{(1)}$, and $V$ is a new gauge field on the lattice $T^{(1)}$. The averaging operation is defined by the formula $(0.4)$, or $(0.12)$ in $[\mathrm{I}]$, but we may take any averaging operation satisfying several general properties, analogous to the properties (0.5)-(0.9) [I]. We have to apply an additional operation after each renormalization transformation $T$. This operation is denoted by $\mathbf{R}$, and it changes effective densities on large field regions. Thus, we construct a sequence of effective densities $\left\{\varrho_{k}\right\}$ by applying successively the operations $\mathbf{R} T$ to the initial density $\varrho_{0}=\exp \left[-\left(1 / g_{0}^{2}\right) A-E\right]$, where $A$ is the Wilson action, and $E$ is a normalization

\footnotetext{
* Research supported in part by the Air Force under Grant AFOSR-86-0229 and by the National
} Science Foundation under Grant DMS-86-02207 
constant. We have

$$
\varrho_{k}=\mathbf{R} T \varrho_{k-1}=(\mathbf{R} T)^{k} \varrho_{0},
$$

and we finish the inductive procedure when we reach the unit lattice. All the details of the procedure, in particular all geometric definitions, are summarized in the Introduction in [I], and in previous papers, references to which can be found in [I]. To describe the effective densities $\varrho_{k}$, and to understand their properties, we apply a combination of the methods and the representations of the papers [16], [I]. The numbers here mean the numbers in the references to [I]. Basic aspects of the procedure are the same as in the paper [16], i.e., we do the integration, and all the other operations, in the small field regions only, but there are two fundamental differences. The first is, that now we are interested not in bounds, but in a precise description of the effective densities in the form of convergent expansions, so we apply the whole analysis of the paper [I] to expressions in the small field regions. The second difference is that we apply the additional operation $\mathbf{R}$, which changes expressions connected with the large field regions. Let us explain briefly why it is necessary to make such changes, or why it is necessary to renormalize the large field expressions, and what is the general structure of the operation $\mathbf{R}$. Consider a large plaquette variable in the first step. The restrictions on these variables are the same as in [16], so we have $|U(\partial p)-1| \geqq g_{0} p_{0}\left(g_{0}\right)$ for a plaquette $p \in T_{1}$, where $p_{0}\left(g_{0}\right)=A_{0}\left(\log g_{0}^{-2}\right)^{p_{0}}$ with a positive integer $p_{0}$. The term in the Wilson action, corresponding to the plaquette $p$, gives the estiomate

$$
\exp \left[-\frac{1}{g_{0}^{2}}[1-\operatorname{Retr} U(\partial p)]\right] \leqq \exp \left(-\frac{1}{2} p_{0}^{2}\left(g_{0}^{2}\right)\right) \leqq g_{0}^{A_{0}\left(\log g_{0}^{-2}\right) p_{0}-1}
$$

For $d<4$ we have $g_{0}=g \varepsilon^{1 / 2(4-d)}$, and the bound above can be estimated by an arbitrarily large power of $\varepsilon$. This is enough to control expressions arising in the large field regions surrounding the plaquette $p$ for all steps of the procedure, i.e., until we reach the unit lattice. For $d=4$ the bare coupling constant behaves asymptotically as $\left(a+b \log \varepsilon^{-1}\right)^{-1 / 2}$, for $\varepsilon \rightarrow 0$, with some positive constants $a, b$, hence the bound does not give any positive power of $\varepsilon$. It is still small for $\varepsilon$ small, and it controls a large number of steps, but this number is a small fraction of the total number of steps. Thus, for some large field regions there is a difficulty in continuing the procedure of [16], the small factor arising from large fields in this region does not control further steps. In such situations we have to change the procedure in order to improve the small factor, i.e., we have to be able to renormalize the expression corresponding to the large field region.

The operation $\mathbf{R}$ serves this purpose. We will not describe it here, we will only assume that it has some properties incorporated in the inductive description of the effective actions.

Let us explain now what is the basic result of this paper. We give a detailed and precise description of the effective densities $\varrho_{k}$ in terms of convergent expansions, and we show that $T \varrho_{k}$ satisfy the same assumptions. This description is quite complicated and technical, and it occupies all of Sect. 2, so here we can formulate only a theorem, or rather an idea of a theorem, referring to the later description. Thus we have 
Theorem. If $\varrho_{k}$ satisfies the assumptions described in detail in Sect. 2, then $T \varrho_{k}$ satisfies also the corresponding assumptions.

This whole paper gives a proof of this, and related theorems. Let us make few comments about the proof, and the presentation. We apply the rule that if some aspect of the procedure is discussed in one situation, this discussion is not repeated in other situations, only changes and modifications. For example, in paper [I] we have discussed in detail the renormalization of the effective actions, and the localization operation for terms of the actions. Here we do not repeat these discussions; although we need the results of the operations in many places, we describe only in each case necessary changes. Generally, we discuss in this paper new issues only; thus we describe in detail all operations connected with introductions of characteristic functions for field variables. Many new characteristic functions appear in connection with large field regions, and they play an important, although quite technical, role in the procedure.

Thus, the papers [I, II] are an essential part of the whole analysis, and the results obtained here are based on the results of those papers. In fact, the analysis of expressions connected with small field regions is the most important part of the method, and even analyzing the $R$-operations in the future we will consider only small field parts of the corresponding large field regions, and we perform operations in this part only.

\section{The First Renormalization Transformation}

We analyze the integral defining the first renormalization transformation (0.1), mainly to understand some basic features of the inductive assumption which will be formulated in the next section. Many of the operations described here are common for all steps of the renormalization procedure, and we will mention them only briefly in the sequel.

At first we introduce two additional partitions of the lattice $T_{1}$ into large cubes. The first is a partition into cubes of the size $M R_{0}$, where $R_{0}$ is the smallest power of $L$ such that $R_{0} \geqq\left(\log g_{0}^{-2}\right)^{r}, r \geqq 2$, and $M=L^{m}$ is sufficiently large. The second is a partition into cubes of the size $L M_{2} R_{0}$, where $M_{2}=L^{m_{2}}$, and $M_{1}<M_{2}<M$. Let us recall that $M_{1}$ is the size of large cubes for which all the theorems of the previous papers, especially those concerning the variational problem, are valid. We fix $M_{2}$ not too much larger that $M_{1}$, e.g., we can take $M_{2}=L^{2} M_{1}$, and $M$ will be chosen much larger than $M_{2}$. The choice will be dictated by many conditions involving this constant. The above partitions have to be compatible, and compatible with the partition $\pi_{0}$ as well, and with other partitions introduced later. The cubes of the second partition are denoted by $\square$. The operation applied to these cubes means adding one layer of $M_{2} R_{0}$-cubes touching $\square$. Usually this operation applied to other sets means adding one layer of cubes taken from this family of cubes, in terms of which the sets are defined.

Now we introduce the decomposition of unity, similar to the decomposition (7) in [16]: 


$$
\begin{aligned}
1 & =\sum_{P_{0}} \prod_{\square \subset P_{0}^{c}} \chi\left(\left\{\sup _{p \subset \square^{-}}|U(\partial p)-1|<\varepsilon_{0}\right\}\right) \prod_{\square \subset P_{0}} \chi\left(\left\{\sup _{p \subset \square-}|U(\partial p)-1| \geqq \varepsilon_{0}\right\}\right) \\
& =\sum_{P_{0}} \chi_{0}\left(P_{0}^{c}\right) \chi_{0}^{c}\left(P_{0}\right),
\end{aligned}
$$

where the sum is over sets $P_{0}$, which are unions of the cubes of the second partition introduced above, i.e., unions of the $L M_{2} R_{0}$-cubes. There are two convenient choices of the positive constant $\varepsilon_{0}$. The first is the same as in [16], i.e., in the first step we take $\varepsilon_{0}=g_{0} p_{0}\left(g_{0}\right), p_{0}\left(g_{0}\right)=A_{0}\left(\log g_{0}^{-2}\right)^{p_{0}}, p_{0} \geqq 5 r$ and $A_{0}$ is a sufficiently large constant. The second is obtained by replacing $g_{0}^{-1}$ in the logarithmic factor $p\left(g_{0}\right)$ by its upper bound coming from the inequality $(0.33)$ [I], i.e., we take $\varepsilon_{0}$ $=g_{0} A_{0}\left(\log \gamma_{0}^{-2}\right)^{p_{0}}, \gamma_{0}^{-2}=\gamma^{-2}+\beta \log \varepsilon^{-1}, \beta, \gamma>0$. The first choice is simpler, and more convenient to study connections with perturbation expansions. The second choice is more convenient in some nonperturbative aspects of the procedure, but it is technically more difficult and we assume here that $\varepsilon_{0}$ is given by the first formula. If a plaquette $p^{\prime}$ is contained in $\left(P_{0}^{c}\right)^{(1)}$, then Proposition 1 [12] implies that the new field $V$ satisfies the bound $\left|V\left(\partial p^{\prime}\right)-1\right|<2 L^{2} \varepsilon_{0}$. We introduce stronger restrictions on $V$ by a next decomposition of unity. We introduce the next two partitions of the lattice $T_{1}$. The first is into cubes of the size $L M R_{1}$, where $R_{1}$ is the smallest power of $L$ such, that $R_{1} \geqq\left(\log g_{1}^{-2}\right)^{r}$. The second is into cubes of the size $L^{2} M_{2} R_{1}$. For the more natural scale $L^{-1}$ these are partitions of the lattice $T_{L^{-1}}$ into $M R_{1}$-cubes and $L M_{2} R_{1}$-cubes correspondingly. Denote by $P_{0}^{\prime}$ the union of the $L M R_{1}$-cubes intersecting $P_{0}$, and take $P_{0}^{\prime \sim}$ (the operation $\sim$ is determined by the $L M R_{1}$-cubes). For every $L^{2} M_{2} R_{1}$-cube $\square^{\prime}$ contained in $\left(P_{0}^{\prime \sim}\right)^{c}$ we construct the function $U_{1, \square^{\prime}}(V)$ as

$$
U_{1, \square^{\prime}}(V)=U\left(\mathbf{B}_{1}\left(\square^{\prime \sim 4}\right), M^{*}\left(Q_{1}^{s^{*}} V\right)\right)
$$

where $\mathbf{B}_{1}\left(\square^{\prime \sim 4}\right)$ is the minimal determining set based on $\square^{\prime \sim 4}$, and $Q_{1}^{s^{* 4}} V$ was introduced in (4.5.3) [18]. Let us recall the definition

$$
\left(Q_{1}^{s^{*}} V\right)(b)=\left\{\begin{array}{lll}
1 & \text { for } & b \subset B(y), \quad y \in T^{(1)} \\
V(c) & \text { for } & b \in B(c)=\left\{b: b_{-} \in B\left(c_{-}\right), b_{+} \in B\left(c_{+}\right)\right\}, \quad c \in T^{(1)} .
\end{array}\right.
$$

The function in (1.2) depends on the field $V$ restricted to $\square^{\prime \sim 4}$. Now, the next decomposition of unity is

$$
\begin{aligned}
1= & \sum_{P_{1}} \prod_{\square^{\prime} \subset P_{\mathrm{f}}} \chi\left(\left\{\sup _{p \subset \square^{\prime} \sim}\left|U_{1, \square^{\prime}}(V, \partial p)-1\right|<\varepsilon_{1} L^{-2}\right\}\right) \\
& \cdot \prod_{\square^{\prime} \subset P_{1}} \chi\left(\left\{\sup _{p \subset \square^{\prime} \sim}\left|U_{1, \square^{\prime}}(V, \partial p)-1\right| \geqq \varepsilon_{1} L^{-2}\right\}\right)=\sum_{P_{1}} \chi_{1}\left(P_{1}^{c}\right) \chi_{1}^{c}\left(P_{1}\right),
\end{aligned}
$$

where the sum is over sets $P_{1} \subset\left(P_{0}^{\prime \sim}\right)^{c}$, which are unions of the $L^{2} M_{2} R_{1}$-cubes, $P_{1}^{c}$ denotes the complement to $\left(P_{0}^{\prime \sim}\right)^{c}$, and $\varepsilon_{1}=g_{1} p_{0}\left(g_{1}\right)$.

Denote by $P_{1}^{\prime}$ the union of the $L M R_{1}$-cubes intersecting $P_{1}$, and denote $P_{1}^{1}$ $=\left(\left(P_{0}^{\prime \sim}\right)^{c} \cap\left(P_{0}^{\prime \sim 2}\right)^{c}\right)^{(1)}$. In blocks of $B\left(P_{1}^{1}\right)$ we introduce the axial gauge fixing expression, the same as in $(0.16)[I]$. Thus we use the identity 


$$
1=\prod_{y \in P_{1}^{1}} \prod_{\substack{x \in B(y) \\ z \neq y}} \int d u(x) \frac{1}{z} \chi\left(\left\{\left|U^{u}(y, x)-1\right|<\varepsilon_{0}\right\}\right) \exp \left[-\frac{1}{g_{0}^{2}}\left[1-\operatorname{Retr} U^{u}(y, x)\right]\right],
$$

where $U(y, x)$ are the variables introduced by $(0.11)$ [I]. We insert it under the integral, and we apply the Faddeev-Popov procedure. This yields the equality

$$
\begin{aligned}
\varrho_{1}(V)= & \sum_{P_{0} P_{1}} \chi_{1}^{c}\left(P_{1}\right) \chi_{1}\left(P_{1}^{c}\right) \int d U \delta\left(\bar{U} V^{-1}\right) \chi_{0}^{c}\left(P_{0}\right) \chi_{0}\left(P_{0}^{c}\right) \chi_{A x}\left(P_{1}^{1}\right) \\
& \cdot \exp \left[-\frac{1}{g_{0}^{2}} \mathbf{G}\left(P_{1}^{1}, U\right)-\frac{1}{g_{0}^{2}} A(U)-\left(L^{4}-1\right)\left|P_{1}^{1}\right| \log z-E\right]
\end{aligned}
$$

where

$$
\mathbf{G}\left(P_{1}^{1}, U\right)=\sum_{y \in P_{1}^{1}} \sum_{\substack{x \in B(y) \\ x \neq y}}[1-\operatorname{Retr} U(y, x)] .
$$

Let us make a remark about the gauge fixing. The main point in introducing it in the form (1.5) is to preserve the Euclidean invariance, but this can be done also with the gauge fixing defined by the $\delta$-functions of the contour variables $U\left(\Gamma_{y, x}\right)$, as in [16] and in the previous papers. For a chosen coordinate system in a block we fix the gauge introducing the $\delta$-functions, as in (9), (10) [16], and we average over the Euclidean transformations of the lattice, leaving the center of the block invariant. This definition has all the properties necessary for the method presented in this paper, in particular it is localizable in subdomains of the lattice, so it can be an alternative to the above definition.

Trying to imitate the procedure in the paper [I] as closely as possible we should introduce now restrictions on a fluctuation field. This field is not defined yet, therefore we introduce restrictions on an approximate fluctuation field. A good approximation on a cube $\square^{\prime} \subset B\left(P_{1}^{1}\right)$ is given by $U U_{1, \square^{\prime}}^{-1}$, where $U_{1, \square^{\prime}}$ is taken in the axial gauge. It is easy to see, by the same reasoning as in the proof of Lemma 1 [14], that $\left|U U_{1, \square^{\prime}}^{-1}-1\right|<O\left(L^{2}\right) \varepsilon_{0}$ on $\square^{\prime 2}$. A domain of this type, with $O\left(L^{2}\right)$ replaced by a small constant, is also contained in the domain of integration in (1.6). These remarks serve as a justification of the following decomposition of unity:

$$
\begin{aligned}
1= & \sum_{Q_{1}} \prod_{\square^{\prime} \subset Q_{1}^{c}} \chi\left(\left\{\sup _{b \in\left(\square^{\prime} \sim^{2}\right)^{*}}\left|U(b) U_{1, \square^{\prime}}^{-1}(b)-1\right|<2 \delta_{0}\right\}\right) \\
& \cdot \prod_{\square^{\prime} \subset Q_{1}} \chi\left(\left\{\sup _{b \in\left(\square^{\prime} \sim^{2}\right)^{*}}\left|U(b) U_{1, \square^{\prime}}^{-1}(b)-1\right| \geqq 2 \delta_{0}\right\}\right)=\sum_{Q_{1}} \chi_{0}^{\prime}\left(Q_{1}^{c}\right) \chi_{0}^{\prime c}\left(Q_{1}\right),
\end{aligned}
$$

where the sum is over sets $Q_{1} \subset\left(B\left(P_{1}^{1}\right)\right)^{\sim-1}=\left(\left(B\left(P_{1}^{1}\right)^{c}\right)^{\sim}\right)^{c}$, which are unions of the $L^{2} M_{2} R_{1}$-cubes, and $\delta_{0}=g_{0} A_{1} p_{0}\left(g_{0}\right)=\left(A_{1} / A_{0}\right) \varepsilon_{0}$. We assume that the constant $A_{0}$ is much larger than $A_{1}$. The superscript * used in (1.8) means that we take the set of all bonds with at least one end-point belonging to $\square^{\prime \sim 2}$, except the bonds $b_{0}(c)$ for $c$, such that at least one end-point belongs to $\left(\square^{\prime \sim 2}\right)^{(1)}$. More generally, for a set $X$, which is a union of unit blocks, we define

$$
X^{*}=X \backslash\left\{b_{0}(c): c \in X^{(1)}\right\} \text {. }
$$


We take $Q_{1}^{\prime}$ again as the union of $L M R_{1}$-cubes which intersect $Q_{1}$, and we surround the sets $Q_{1}^{\prime},\left(B\left(P_{1}^{1}\right)^{c}\right)^{\sim}$ by two layers of $L M R_{1}$-cubes. Denote

$$
\Omega_{1}=\left(Q_{1}^{\prime \sim 2} \cup\left(B\left(P_{1}^{1}\right)^{c}\right)^{\sim 3}\right)^{c}=\left(Q_{1}^{\prime \sim 2}\right)^{c} \cap\left(B\left(P_{1}^{1}\right)\right)^{\sim-3}
$$

thus $\Omega_{1}$ is a union of $L M R_{1}$-cubes, and a distance between $\Omega_{1}$ and the union of the large field regions is at least $2 L M R_{1}$. On the set $\Omega_{1}^{\sim}$ we have only the small field characteristic functions, and it is easy to see that the functions $\chi_{0}, \chi_{A x}$ localized in $\Omega_{1}^{\sim}$ are equal to 1 , if $A_{0}$ is sufficiently larger than $A_{1}$. We drop these functions from the integrals in (1.6).

We fix $\Omega_{1}$ and we do the resummation with respect to $P_{0}, P_{1}, Q_{1}$, as in (8) [16]. This yields the equality

$$
\begin{aligned}
\varrho_{1}(V)= & \sum_{\Omega_{1}} \chi_{1}\left(\Omega_{1}\right) \int d U \delta\left(\bar{U} V^{-1}\right) \zeta\left(\Omega_{1}^{c}\right) \chi_{0}^{\prime}\left(\Omega_{1}\right) \\
& \cdot \exp \left[-\frac{1}{g_{0}^{2}} \mathbf{G}\left(\Omega_{1}^{(1)}, U\right)-\frac{1}{g_{0}^{2}} A(U)-\left(L^{4}-1\right)\left|\Omega_{1}^{(1)}\right| \log z-E\right] .
\end{aligned}
$$

Here the factor $\zeta\left(\Omega_{1}^{c}\right)$ is defined by all characteristic functions and gauge fixing exponential factors localized in $\Omega_{1}^{c}$, and resummed over all admissible $P_{0}, P_{1}, Q_{1}$.

The next step is again the same as in Sect. A of [16], we make the translation $U$ $=U^{\prime} U_{1}$ in the integral in (1.11). Let us denote by $V_{0}$ the integration variables localized in $\Omega_{1}^{c}$, i.e. $V_{0}=\left.U\right|_{\Omega_{1}^{c}}$. The configuration $U_{1}$, or more precisely $U_{1}\left(\Omega_{1}, V\right)$, is defined as the critical point of the functional

$$
U \rightarrow A(U)+\mathbf{G}\left(\Omega_{1}^{(1)}, U\right) \quad \text { for } \quad U: U=V_{0} \quad \text { on } \quad \Omega_{1}^{c}, \quad \bar{U}=V \quad \text { on } \quad \Omega_{1}^{(1)} .
$$

It is equal to the critical point of the action $A(U)$ only, on the subspace of configurations satisfying the above conditions and the axial gauge conditions $U(y, x)=1$ for $x \in B(y), x \neq y, y \in \Omega_{1}^{(1)}$. By Theorem 1 [15] there exists exactly one critical point of (1.12) in the domain of integration in (1.11), for $\varepsilon_{0}$ sufficiently small. It is a minimum of (1.12), with a strictly positive second order differential. The field $U^{\prime}$ is small, because $U^{\prime}=U U_{1}^{-1}=\left(U U_{1, \square^{\prime}}^{-1}\right)\left(U_{1, \square^{\prime}} U_{1}^{-1}\right)$, and both fields in the parenthesis on the right-hand side of this equality are small. We denote

$$
A^{\prime}=\frac{1}{i} \log U^{\prime}, \quad \text { or } \quad U^{\prime}=\exp i A^{\prime}
$$

and we call $A^{\prime}$ a fluctuation field.

Now we expand the expressions in the integral (1.11) with respect to the fields $A^{\prime}$. This expansion was investigated in [15], see Sect. B. The formula (26) there gives an expansion of the action, see also (I.2.7). An expansion of the gauge fixing terms is given by (I.1.8). For the expressions under the $\delta$-functions we have (14)-(16) [16], or (I.1.6), (I.1.7) restricted to $\Omega_{1}^{(1)}$. We linearize these expressions by the transformations analyzed between (16)-(18) [16], or between (I.1.8)-(I.1.9) [I]. These 
operations yield

$$
\begin{aligned}
\varrho_{1}(V)= & \left.\sum_{\Omega_{1}} \chi_{1}\left(\Omega_{1}\right) \int d V_{0}\right|_{\Omega_{1}^{c}} \delta\left(\bar{V}_{0} V^{-1}\right) \zeta\left(\Omega_{1}^{c}\right) \\
& \cdot \exp \left[-\frac{1}{g_{0}^{2}} A\left(U_{1}\right)+\left|\Omega_{1}^{*}\right| \log \sigma_{0}-\left(L^{4}-1\right)\left|\Omega_{1}^{(1)}\right| \log z-E\right] \\
& \left.\cdot \int d A\right|_{\Omega_{1}} \delta(\widetilde{Q} A) \chi_{0}^{\prime}\left(\Omega_{1}\right) \exp [\log \sigma(A-h \widetilde{D}(A)) \\
& +\operatorname{Tr} \log \left(I-h\left(\frac{\delta}{\delta A} \widetilde{D}\right)(A)\right)-\frac{1}{g_{0}^{2}} \mathbf{G}(A-h \widetilde{D}(A)) \\
& -\frac{1}{g_{0}^{2}}\left\{\left\langle A-h \widetilde{D}(A), J_{1}\right\rangle+\frac{1}{2}\langle A-h \widetilde{D}(A), \Delta(A-h \widetilde{D}(A))\rangle\right. \\
& \left.\left.+V_{0}(A-h \widetilde{D}(A))\right\}\right] .
\end{aligned}
$$

Let us recall that the linearizing transformation is different from the identity only on bonds $\left\{b_{0}(c): c \in \Omega_{1}^{(1)}\right\}$, hence the characteristic function $\chi_{0}^{\prime}\left(\Omega_{1}\right)$, defined in (1.8), is unchanged under the transformation. The number $\left|\Omega_{1}^{*}\right|$ is the number of bonds belonging to $\Omega_{1}$ minus the number of bonds in the set $\left\{b_{0}(c): c \in \Omega_{1}^{(1)}\right\}$. Also, let us recall that the functions and operators on the right-hand side of (1.14) depend on the configuration $U_{1}$. Now we perform the scaling transformation $A=g_{0} A^{\prime}$ in the integral with respect to variables $A$, and we remove the $\delta$-functions under the integral solving the equations $\left(\widetilde{Q} A^{\prime}\right)(c)=0$ on $\Omega_{1}^{(1)}$. The equation determines the variable $A^{\prime}\left(b_{0}(c)\right)$ as a linear function of the remaining variables $A^{\prime}(b)$, $b \subset B\left(c_{-}\right) \cup B\left(c_{+}\right), b \neq b_{0}(c)$. We denote this function by $C$, and we denote by $A$ the integration variables restricted to bonds $\Omega_{1}^{*}=\Omega_{1} \backslash\left\{b_{0}(c): c \in \Omega_{1}^{(1)}\right\}$. Hence $A^{\prime}=C A$ are solutions of the above equations and we have $\widetilde{Q} C A=0$. After these operations we obtain

$$
\begin{aligned}
\varrho_{1}(V)= & \left.\sum_{\Omega_{1}} \chi_{1}\left(\Omega_{1}\right) \int d V_{0}\right|_{\Omega_{1}^{c}} \delta\left(\bar{V}_{0} V^{-1}\right) \zeta\left(\Omega_{1}^{c}\right) \\
& \cdot \exp \left[-\frac{1}{g_{0}^{2}} A\left(U_{1}\right)+\log g_{0} d(\mathbf{g})\left|\Omega_{1}^{*}\right|+\log \sigma_{0}\left|\Omega_{1}^{*}\right|-\log z\left(L^{4}-1\right)\left|\Omega_{1}^{(1)}\right|-E\right] \\
& \left.\cdot z^{(0)} \int d A\right|_{\Omega_{1}} \chi_{0}^{\prime}\left(\Omega_{1}\right) \exp \left[-\frac{1}{2}\left\langle A, C^{*} \Delta_{1} C A\right\rangle+v\left(g_{0} C A\right)-\frac{1}{g_{0}^{2}} V\left(g_{0} C A\right)\right],
\end{aligned}
$$

where

$$
\left\langle A, \Delta_{1} A\right\rangle=\langle A, \Delta A\rangle-2\left\langle h C^{(2)}(A), J_{1}\right\rangle+\mathbf{G}^{(2)}(A),
$$

and $v(A), V(A)$ have the same meaning as in (27) [16], i.e. $v(A)$ is the sum of the first two terms under the second exponential in (1.15), and $V(A)$ is the sum of the remaining terms of the order higher than 2 . The factor $z^{(0)}$ is obtained by integrating the $\delta$-functions with respect to the variables $A\left(b_{0}(c)\right), c \in \Omega_{1}^{(1)}$. 
Let us remark that it is very important for our method, as it was in [16, I], that we expand in (1.14) around the critical point, so the potentially dangerous linear term $\left(1 / g_{0}^{2}\right)\left\langle A, J_{1}\right\rangle$ vanishes on the domain of integration. Another important remark is connected with gauge invariance. As in all previous papers the expressions in (1.14)-(1.16), except the characteristic function $\chi_{0}^{\prime}\left(\Omega_{1}\right)$, are invariant with respect to the simultaneous gauge transformations

$$
\begin{aligned}
& U_{1} \rightarrow U_{1}^{u}, \quad U_{1}^{u}\left(x, x^{\prime}\right)=u(x) U_{1}\left(x, x^{\prime}\right) u^{-1}\left(x^{\prime}\right), \\
& A \rightarrow R(u) A, \quad(R(u) A)\left(x, x^{\prime}\right)=R(u(x)) A\left(x, x^{\prime}\right)=u(x) A\left(x, x^{\prime}\right) u^{-1}(x),
\end{aligned}
$$

where $u$ is a $G$-valued function defined on sites of the lattice $T$. The characteristic function is invariant with respect to gauge transformations of the new field $V: V \rightarrow V^{v}, v$ is a $G$-valued function defined on sites of the new lattice $T^{(1)}$. Such a transformation $v$ determines the gauge transformation $u_{0}, u_{0}(x)=v(y)$ for $x \in B(y)$, and the field $U_{0}$ constructed in (1.6) transforms by $u_{0}: U_{0} \rightarrow U_{0}^{u_{0}}$. This implies that the characteristic function $\chi_{0}^{\prime}\left(\Omega_{1}\right)$ is invariant if the field variables $A$ are transformed by the adjoint representation $A \rightarrow R\left(u_{0}\right) A$. In the future we will use both invariance properties : the invariance of all the expressions determining the density with respect to the gauge transformations of the new field, and the invariance of the effective action with respect to the gauge transformations (1.17). To get this invariance for the last integral in (1.16) we have to replace the characteristic function $\chi_{0}^{\prime}\left(\Omega_{1}\right)$ by functions depending on $A$ only. We introduce a new decomposition of unity in the domain $\Omega_{1}^{\sim-1}=\left(\left(\Omega_{1}^{c}\right)^{\sim}\right)^{c}$ :

$$
\begin{aligned}
1= & \sum_{R_{1}} \prod_{\square^{\prime} \subset R_{1}^{c}} \chi\left(\left\{\sup _{b \in\left(\square^{\prime} \sim 2\right)^{*}}|A(b)|<g_{0}^{-1} \delta_{0}\right\}\right) \\
& \cdot \prod_{\square^{\prime} \subset R_{1}} \chi\left(\left\{\sup _{b \in\left(\square^{\prime} \sim 2\right)^{*}}|A(b)| \geqq g_{0}^{-1} \delta_{0}\right\}\right)=\sum_{R_{1}} \chi^{(o)}\left(R_{1}^{c}\right) \chi^{(0) c}\left(R_{1}\right) .
\end{aligned}
$$

Here the summation is over sets $R_{1} \subset \Omega_{1}^{\sim-1}$, which are unions of $L^{2} M_{2} R_{1}$-cubes, and $R_{1}^{c}$ means the complement to $\Omega_{1}^{\sim}{ }^{-1}$. Consider a cube $\square^{\prime} \subset R_{1}^{c}$. For this cube we now have the product of the two characteristic functions,

$$
\begin{aligned}
& \chi\left(\left\{\sup _{b \in\left(\square^{\prime} \sim 2\right)^{*}}|A(b)|<g_{0}^{-1} \delta_{0}\right\}\right) \\
& \quad \chi \chi\left(\left\{\sup _{b \in\left(\square^{\prime} \sim 2\right)^{*}}\left|\exp i g_{0} A(b) U_{1}(b) U_{1, \square^{\prime}}^{-1}(b)-1\right|<2 \delta_{0}\right\}\right) .
\end{aligned}
$$

The cube $\square^{\prime \sim 4}$ is contained in $\Omega_{1}$, and both configurations $U_{1}, U_{1, \square^{\prime}}$ are determined by the same configuration $V$ on $\square^{\prime \sim 3}$, hence on $\square^{\prime \sim 2}$ the difference between these configurations is very small. More precisely $\left|U_{1} U_{1, \square^{\prime}}^{-1}-1\right|<O$ (1) $B_{3}$ $\cdot \exp \left(-\delta L M_{2} R_{1}\right) \varepsilon_{1}$, and the bound can be made much smaller than $\delta_{0}$, if $M_{2} R_{1}$ is large enough. Thus the second function in the product (1.19) is equal to 1 , and we can omit it. These functions remain only for $\square^{\prime} \subset\left(\Omega_{1} \backslash \Omega_{1}^{\sim^{-1}}\right) \cup R_{1}$, more precisely they are the only functions for $\square^{\prime} \subset \Omega_{1} \backslash \Omega_{1}^{\sim-1}$, and for $\square^{\prime} \subset R_{1}$ they are multiplied by the corresponding large field characteristic function in (1.18). We denote again by 
$R_{1}^{\prime}$ the union of $L M R_{1}$-cubes intersecting the set $R_{1}$, and we introduce the sets

$$
\Lambda_{1}=\Omega_{1}^{\sim-2} \cap\left(R_{1}^{\prime \sim}\right)^{c}, \quad S_{1}=\left(\Omega_{1} \backslash \Omega_{1}^{\sim-1}\right) \cup R_{1} .
$$

On the set $\Lambda_{1}^{\sim}$ we have only the small fluctuation field characteristic functions from (1.18). We make an additional small modification of the domain $\Lambda_{1}$. The complement $\Lambda_{1}^{c}$ is a union of connected components, and some of them may be relatively small. We distinguish components which are contained in cubes of sizes smaller than $100 L M R_{1}$, and we replace such components by the smallest rectangular parallelepipeds containing them. Such a rectangular parallelepiped is the smallest convex domain, which is a union of $L M R_{1}$-cubes, containing a given component. This change is insignificant from the point of view of bounds, but it simplifies some geometric aspects of the large field problem, and the R-operation. This domain $\Lambda_{1}$ is the final small field domain, and we will perform the conditional integration in the integral (1.15) with respect to the fluctuation field $A$ restricted to $\Lambda_{1}$.

The characteristic functions connected with $S_{1}$ involve the onfiguration $U_{1}$, therefore they depend on the new field $V$ on the whole small field domain $\Lambda_{1}$. This is an undesirable situation for the next renormalization step, and now we will remove this dependence. Introduce the function $U_{1}\left(\Omega_{1} \cap \Lambda_{1}^{c}, V\right)$, which is determined by the same conditions on the domain $\left(\Lambda_{1}^{\sim}\right)^{c}$ as the function $U_{1}\left(\Omega_{1}, V\right)$, and on the domain $\Lambda_{1}^{\sim} \backslash \Lambda_{1}$ it is determined by the set $\mathbf{B}_{1}\left(\Lambda_{1}^{c}\right)$ and the configuration $Q_{1}^{s^{*}} V$. This function depends on $V$ restricted to $\Omega_{1} \cap \Lambda_{1}^{c}$. We have

$$
\begin{aligned}
U_{1} & =U_{1}\left(\Omega_{1} \cap \Lambda_{1}^{c}, M^{*}\left(U_{1}\right)\right) \\
& =\exp i L^{-1} \mathbf{H}_{1, A x}\left(\Omega_{1} \cap \Lambda_{1}^{c}, \frac{1}{i} \log \left[M^{\cdot}\left(U_{1}\right)\left(M^{\cdot}\left(Q_{1}^{s^{*}} V\right)\right)^{-1}\right]\right) U_{1}\left(\Omega_{1} \cap \Lambda_{1}^{c}, V\right),
\end{aligned}
$$

where the function $\mathbf{H}_{1, A x}$ depends on its argument restricted to a small neighborhood of $\partial \Lambda_{1}$ (in fact one layer of $M_{1}$-cubes touching $\partial \Lambda_{1}$ and contained in $\Lambda_{1}^{c}$ ). The dependence is analytic, and the function $\mathbf{H}_{1, A x}$ has the exponential decay property. It was formulated in (190) Sect. G of [15] for the Landau gauge, but on the unit lattice it holds for any gauge, for which propagators are exponentially decaying, hence for the axial gauge too. This implies that the function $H_{1, A x}$ is very small on $\square^{\prime}$, for $\square^{\prime} \subset S_{1}$, because the distance between $\square^{\prime}$ and the support of the argument is greater than $M R_{1}$. More precisely, we have $\left|\mathbf{H}_{1, A x}\right|<O(1) B_{3} \exp \left(-\delta M R_{1}\right) \varepsilon_{1}$, and the bound is much smaller than $\delta_{0}$, for $M R_{1}$ large enough. Now we make a change of variables for each bond variable $A(b), b \in S_{1}^{*}$. There are two forms of this change of variables. For $b \in\left(R_{1}^{\sim 2} \cap S_{1}\right)^{*}$, we take

$$
\begin{aligned}
A(b)= & \frac{1}{i g_{0}} \log \exp i g_{0} A^{\prime}(b) \exp \left(-i g\left(A^{\prime}(b)\right) \mathbf{H}_{1, A x}(b)\right) \\
= & A^{\prime}(b)-\frac{1}{g_{0}} g\left(A^{\prime}(b)\right) \mathbf{H}_{1, A x}(b)-\frac{1}{g_{0}} \mathbf{F}\left(g_{0} A^{\prime}(b),\right. \\
& \left.-g\left(A^{\prime}(b)\right) \mathbf{H}_{1, A x}(b)\right) .
\end{aligned}
$$


Here $g$ is a $C^{\infty}$-function defined on the Lie algebra $\mathbf{g}, 0 \leqq g\left(A^{\prime}\right) \leqq 1, g\left(A^{\prime}\right)=0$ on $\left\{A^{\prime} \in \mathbf{g}:\left|A^{\prime}\right| \leqq 4 / 3 g_{0}^{-1} \delta_{0}\right\}, g\left(A^{\prime}\right)=1$ on $\left\{A^{\prime} \in \mathbf{g}:\left|\exp i g_{0} A^{\prime}-1\right| \geqq 5 / 3 \delta_{0}\right\}$. The configuration $U_{1}\left(\Omega_{1} \cap \Lambda_{1}^{c}\right) U_{1}^{-1} \square^{\prime}$, is very small on $\square^{\prime \prime 2}$ in this case, hence $g\left(A^{\prime}\right)=1$ on a neighborhood of $\left\{A^{\prime} \in \mathbf{g}:\left|\exp i g_{0} A^{\prime} U_{1}\left(\Omega_{1} \cap \Lambda_{1}^{c}\right) U_{1, \square^{\prime}}^{-1}, 1\right| \geqq 2 \delta_{0}\right\}$. The function $\mathbf{F}$ is defined as a sum of terms of orders higher than 1 in the Baker-Campbell-Haussdorf formula (see (29) in [12]). It is at least linear in $g_{0} A^{\prime}(b)$, hence the factor $g_{0}^{-1}$ is cancelled. In the second term on the right-hand side of (1.22) the factor $g_{0}^{-1}$ is suppressed by the bounds on $\mathbf{H}_{1, A x}$. These changes of variables do not change the functions $\chi^{(0)}$, and they transform the function $\chi^{(0) c}\left(\square^{\prime}\right) \chi_{0}^{\prime}\left(\square^{\prime}\right)$ into the function

$$
\begin{aligned}
& \chi\left(\left\{\sup _{b \in\left(\square^{\prime} \sim 2\right)^{*}}\left|A^{\prime}(b)\right| \geqq g_{0}^{-1} \delta_{0}\right\}\right) \\
& \quad \cdot \chi\left(\left\{\sup _{b \in\left(\square^{\prime} \sim 2\right)^{*}}\left|\exp i g_{0} A^{\prime}(b) U_{1}\left(\Omega_{1} \cap \Lambda_{1}^{c}, b\right) U_{1, \square^{\prime}}^{-1}(b)-1\right|<2 \delta_{0}\right\}\right) .
\end{aligned}
$$

For $b \in S_{1}^{*} \backslash\left(R_{1}^{\sim 2}\right)^{*}$ we make a simpler change of variables; we take (1.22) with the function $g\left(A^{\prime}(b)\right)$ replaced by 1 . These changes of variables transform the function $\chi_{0}^{\prime}\left(\square^{\prime}\right)$ into the second factor in the product (1.23). For simplicity we denote the new characteristic functions in the same way as the old ones, and the new variables $A^{\prime}$ by $A$. After these changes of variables the characteristic functions depend on the new variables $V$ restricted to the large field domain $\Lambda_{1}^{c}$. It is the desired localization property.

The change of variables produces new terms in the effective action in (1.15). The Jacobians of the transformations (1.22) can be exponentiated, as in (20) [16], and they yield the expression

$$
\sum_{b \in S_{1}^{*}} \operatorname{Tr} \log \left[1+\frac{\partial}{\partial A(b)}\left(-\frac{1}{g_{0}} g(A(b)) \mathbf{H}_{1, A x}(b)+\frac{1}{g_{0}} \mathbf{F}(\ldots)\right)\right] .
$$

Expanding the terms of the effective action in the second exponential in (1.15) with respect to the last two perturbative terms in (1.22) up to the first order, we obtain a new expression $V^{(0)}\left(S_{1}, A, \mathbf{H}_{1, A x}\right)$. Because of the almost local character of the action in (1.15), it is a sum over bonds $b \in S_{1}$ of the expressions which depend on $A, \mathbf{H}_{1, A x}$ almost locally. The dependence on $\mathbf{H}_{1, A x}$ is analytic, and the expressions are small, i.e., they can be bounded by any positive power of $g_{0}$.

After the change of variables we obtain an expansion of the form (1.15), but with the additional sum over $\Lambda_{0}$, the characteristic function $\chi_{0}^{\prime}\left(\Omega_{1}\right)$ replaced by

$$
\sum_{R_{1}} \chi^{(0)}\left(\Lambda_{1}\right) \chi^{(0)}\left(R_{1}^{c} \cap \Lambda_{1}^{c}\right) \chi^{(0) c}\left(R_{1}\right) \chi_{0}^{\prime}\left(S_{1}\right)
$$

and with the additional term $V^{(0)}\left(S_{1}\right)$ in the effective action. For each term in this expansion consider the conditional integral with respect to the variables $A$ restricted to $\Lambda_{1}$. This integral is equal to the corresponding conditional integral in the case analyzed in [I], i.e. where there are no large field regions, but with a different 
background field. It is given by

$$
\begin{aligned}
& \left.\int d A\right|_{\Lambda_{1}} \chi^{(0)}\left(\Lambda_{1}\right) \exp \left[-\frac{1}{2}\left\langle\Lambda_{1} A, C^{*} \Delta_{1} C \Lambda_{1} A\right\rangle-\left\langle\Lambda_{1}^{c} A, C^{*} \Delta_{1} C \Lambda_{1} A\right\rangle\right. \\
& \left.\quad+v\left(g_{0} C A\right)-\frac{1}{g_{0}^{2}} V\left(g_{0} C A\right)\right] \\
& =Z^{(0)}\left(\Lambda_{1}\right) \int d \mu_{C^{(0)}\left(\Lambda_{1}\right)}(A) \chi^{(0)}\left(\Lambda_{1}\right) \exp \left[-\left\langle\Lambda_{1}^{c} A, C^{*} \Delta_{1} C \Lambda_{1} A\right\rangle+\mathbf{V}^{(0)}\left(g_{0}, A\right)\right] \\
& =\exp \left[\log Z^{(0)}\left(\Lambda_{1}\right)+\frac{1}{2}\left\langle\Lambda_{1}^{c} A, C^{*} \Delta_{1} C C^{(0)}\left(\Lambda_{1}\right) C^{*} \Delta_{1} C \Lambda_{1}^{c} A\right\rangle\right. \\
& \left.\quad+\mathbf{E}^{(1)}\left(\Lambda_{1}, g_{0}, \Lambda_{1}^{c} A\right)\right]
\end{aligned}
$$

The above equalities define the functions $\mathbf{V}^{(0)}$ and $\mathbf{E}^{(1)}$. Let us recall that all the above expressions depend on the configuration $U_{1}$. Of course the main problem is how to represent the function $\mathbf{E}^{(1)}$. We use the exponentiated cluster expansion of [I], but changed properly to yield the more detailed representation we need now, as it was explained in the introductory section. Especially we separate the clusters with localization domains close to $\Lambda_{1}^{c}$, e.g., within the distance $M R_{1}$ to $\Lambda_{1}^{c}$, and we denote the sum of these terms, and the expanded $V^{(0)}\left(S_{1}\right)$, by $\mathbf{B}^{(1)}\left(U_{1}, \Lambda_{1}^{c} A, S_{1}\right)$. This is the boundary term, and it can be bounded by $O(1)\left|\Lambda_{1} \cap \Lambda_{1}^{c}\right|$; therefore it is controlled by the large field estimates, and does not need to be renormalized. This will be discussed thoroughly in the next section.

The final step is renormalization of the effective action. We renormalize it in the region $\Lambda_{1}$ subtracting the values at $U_{1}=1$, and the counterterm $\beta_{1}\left(g_{0}\right) A\left(\phi_{1}, U_{1}\right)$, where $\beta_{1}\left(g_{0}\right)$ was defined in [I] by the formulas (1.41)-(1.43), $\phi_{1} \in C_{0}^{\infty}\left(\Lambda_{1}\right), \phi_{1}=1$ on $\Lambda_{1}^{\tilde{N}^{-1}}$. The symbol $A\left(\phi_{1}, U_{1}\right)$ means that we multiply the term in the Wilson action corresponding to a plaquette $p$ by $\phi_{1}(x(p)), x(p)$ is the initial point of $\partial p$ [i.e., $p=p_{\mu v}(x)$ for some $\mu<v$ ]. Let us write the first renormalization group equation

$$
\frac{1}{g_{0}^{2}}=\frac{1}{g_{1}^{2}(x)}+\beta_{1}\left(g_{0}\right) \phi_{1}(x)
$$

The difference in comparison with Eq. (I.0.20) is that here we have functions on the lattice $T$ instead of the constants there, but on ${\Lambda_{1}^{\sim-1}}^{-1}$ we have the same equation, which defines the same coupling constant $g_{1}$.

To write the final form of the expansion obtained after all these steps, we introduce some new notations. Notice that the set $S_{1}$ determines the set $R_{1}$, and denote the product of all the characteristic functions in (1.25) localized in $\Omega_{1} \cap \Lambda_{1}^{c}$ by $\chi\left(\Omega_{1} \cap \Lambda_{1}^{c}, S_{1}\right)$. Now we can write

$$
\varrho_{1}(V)=\sum_{\left\{\Omega_{1}, \Lambda_{1}\right\}} \chi_{1}\left(\Omega_{1}\right) \mathbf{T}_{1}\left(\left\{\Omega_{1}, \Lambda_{1}\right\}\right) \exp A_{1}\left(\frac{1}{g_{1}^{2}}, U_{1}\right),
$$


where $\mathbf{T}_{1}$ is the integral operation defined by the integration

$$
\begin{aligned}
\mathbf{T}_{1}\left(\left\{\Omega_{1}, \Lambda_{1}\right\}\right)= & \left.\left.\sum_{S_{1}} \int d V_{0}\right|_{\Omega_{1}^{c}} \delta\left(\bar{V}_{0} V^{-1}\right) \zeta\left(\Omega_{1}^{c}\right) \int d A\right|_{\Omega_{1} \cap \Lambda_{1}^{c}} \chi\left(\Omega_{1} \cap \Lambda_{1}^{c}, S_{1}\right) \\
& \cdot \exp \left[-\frac{1}{2}\left\langle A, C^{*} \Delta_{1} C A\right\rangle\right. \\
& \left.+\frac{1}{2}\left\langle A, C^{*} \Delta_{1} C C^{(0)}\left(\Lambda_{1}\right) C^{*} \Delta_{1} C A\right\rangle\right],
\end{aligned}
$$

and the effective action $A_{1}$ has the form

$$
\begin{aligned}
A_{1}\left(\frac{1}{g_{1}^{2}}, U_{1}\right)= & -A\left(\frac{1}{g_{1}^{2}}, U_{1}\right)+\left\{\mathbf{E}^{(1)}\left(\Lambda_{1}, g_{0}, U_{1}\right)-\mathbf{E}^{(1)}\left(\Lambda_{1}, g_{0}, 1\right)\right. \\
& \left.-\beta_{1}\left(g_{0}\right) A\left(\phi_{1}, U_{1}\right)\right\}+\mathbf{B}^{(1)}\left(U_{1}, A, \mathrm{~S}_{1}\right)-E_{1} .
\end{aligned}
$$

Here we have combined the term $\log Z^{(0)}\left(\Lambda_{1}\right)$ with the part of the previous function $\mathbf{E}^{(1)}$ in (1.26), to define the new function $\mathbf{E}^{(1)}$ above. It coincides with the corresponding function constructed in [I], if the last is suitably restricted to the domain $\Lambda_{1}$. This is the reason why we can renormalize it by the same counterterm as in [I], only localized properly. The boundary term $\mathbf{B}^{(1)}$ was described before. The constant $E_{1}$ is obtained from $E$ by subtraction of all the constants which have appeared in the procedure, i.e., the constants in the first exponential in (1.15), and the constant $\mathbf{E}^{(1)}\left(\Lambda_{1}, g_{0}, 1\right)$. Thus $E_{1}$ depends on $\Omega_{1}, \Lambda_{1}$.

The representation (1.28)-(1.30) will be generalized in the next section to an inductive description of the $k$-th effective density, with some modifications connected with new terms obtained by the R-operations.

\section{The Inductive Assumption and Formulations of Results}

We write the inductive assumption for the density obtained after $k$ renormalization transformations. It is written explicitly, as in (1.28)-(1.30) for the first step, except that the operations corresponding to the operation $\mathbf{T}_{1}$ in (1.29) are left undefined, only their basic general properties are formulated. Their inductive definition will follow successively from the constructions of the subsequent section and forthcoming papers.

The $k$-th density $\varrho_{k}\left(V_{k}\right)$ is a function of the new field $V_{k}$ on the lattice $T^{(k)}$. It is represented by an expansion generalizing the expansion (1.28) in the first step. Terms of this expansion are parametrized by sequences of domains in $T$, of the type described already several times in previous papers, e.g. in (1.3)-(1.6) [14], or (38), (39) [16]. Let us start with the description of this geometric setting. We consider sequences of localization domains $\left\{\Omega_{j}\right\}, j=1,2, \ldots, k, \Omega_{j} \in \mathbf{D}_{j}$, such that $\Omega_{1} \supset \Omega_{2} \supset \ldots \supset \Omega_{k}$. For such a sequence we consider sequences of localization domains $\left\{\Lambda_{j}\right\}, \Lambda_{j} \in \mathbf{D}_{j}$, such that

$$
\Omega_{1} \supset \Lambda_{1} \supset \Omega_{2} \supset \Lambda_{2} \supset \ldots \supset \Omega_{j} \supset \Lambda_{j} \supset \ldots \supset \Omega_{k} \supset \Lambda_{k} .
$$

We consider also a third family of sequences $\left\{S_{j}\right\}, j=1,2, \ldots, k$, where $S_{j} \subset \Omega_{j} \cap \Lambda_{j}^{c}$, and $S_{j}$ is either empty, or it is a union of $L M_{2} R_{j}$-cubes in the lattice $T_{L^{-}}$. For the 
domains in (2.1) we admit the possibility that $\Lambda_{j}=\Omega_{j}$ for some indices $j$. Such a situation may arise as a result of an $\mathbf{R}$-operation. If $\Omega_{j} \cap \Lambda_{j}^{c}$ is nonempty, then $S_{j}$ is nonempty too; in fact it contains $\Omega_{j} \backslash \Omega_{j}^{\sim-1}=\Omega_{j} \cap\left(\Omega_{j}^{c}\right)^{\sim}$. There is a characteristic function $\chi\left(\Omega_{j} \cap \Lambda_{j}^{c}, S_{j}\right)$ associated with these domains, which includes a large fluctuation field characteristic function associated with the set $R_{j}=S_{j} \backslash\left(\Omega_{j} \cap\left(\Omega_{j}^{c}\right)^{\sim}\right)$. There is also a function $\zeta\left(\Omega_{j}^{c}\right)$, which includes summations over other large field characteristic functions. These functions were defined in the first step, and their inductive definition will be given in the next section describing the $k+1$-st renormalization step.

For a sequence $\left\{\Omega_{j}\right\}$, where $\Omega_{j}$ are considered as subsets of $T_{\eta}$, we denote

$$
\begin{gathered}
\Gamma_{0}=\Omega_{1}^{c}, \quad \Gamma_{j}=\Omega_{j}^{(j)} \backslash \Omega_{j+1}^{(j)}, \quad j=1, \ldots, k-1, \\
\Gamma_{k}=\Omega_{k}^{(k)}, \quad \text { and } \quad \mathbf{B}=\bigcup_{j=0}^{k} \Gamma_{j} .
\end{gathered}
$$

This definition is meant for sets of points, bonds and plaquettes, with the convention described in Sect. 0 [I]. The set $\mathbf{B}$ determines the sequence $\left\{\Omega_{j}\right\}$, and is called the determining set. The domain $\Omega_{1}$, or rather a small neighborhood of $\Omega_{1}$ including a layer of $M_{1}$-cubes ( $M_{1} \eta$-cubes in the $\eta$-lattice), is called its support. We denote further

$$
Z_{j}=\Lambda_{j}^{c}
$$

These large field regions satisfy the following condition: if a component of $Z_{j}$ is contained in a cube of the size $100 M R_{j}$ (in the $L^{-j}$-lattice), then it is a rectangular parallelepiped.

Now let us define numbers describing sizes of domains and restrictions on fields. We define

$$
\varepsilon_{j}=g_{j} A_{0}\left(\log g_{j}^{-2}\right)^{p_{0}}=g_{j} p_{0}\left(g_{j}\right) ;
$$

$R_{j}$ is the smallest number of the form $L^{r}$ such, that

$$
R_{j} \geqq\left(\log g_{j}^{-2}\right)^{r} .
$$

The coupling constants $g_{j}$ satisfy the inequalities

$$
\begin{aligned}
g_{n} & \leqq\left(1+g_{n}^{2} \beta^{\prime}(n-m)\right)^{1 / 2} g_{m} \leqq\left(1+g_{n}^{2} \beta^{\prime}\right)^{1 / 2}(n-m)^{1 / 2} g_{m} \\
& \leqq\left(1+\beta_{0}\right)(n-m)^{1 / 2} g_{m} \\
g_{m} & \leqq\left(1+\beta_{0}\right) g_{n}
\end{aligned}
$$

where $n>m$, and $\beta_{0}>0$ can be chosen arbitrarily small, if $g$ is sufficiently small. The inequalities follow from the renormalization group equations $(0.20)$ [I], and from the properties of the $\beta$-functions. They imply the following inequalities:

$$
\begin{aligned}
\left(\log g_{n}^{-2}\right)^{p} & \leqq\left(1+\beta_{0}\right)\left(\log g_{m}^{-2}\right)^{p} \\
\left(\log g_{m}^{-2}\right)^{p} & \leqq\left(1+g_{n}^{2} \beta^{\prime}(n-m)\right)^{\beta_{0}}\left(\log g_{n}^{-2}\right)^{p} \\
& \leqq\left(1+\beta_{0}\right)(n-m)^{\beta_{0}}\left(\log g_{n}^{-2}\right)^{p}
\end{aligned}
$$


where $p$ is a positive integer. From the above inequalities we obtain

and

$$
\begin{aligned}
\varepsilon_{n} & \leqq\left(1+\beta_{0}\right)(n-m)^{1 / 2} \varepsilon_{m}, \\
\varepsilon_{m} & \leqq\left(1+\beta_{0}\right)\left(1+g_{n}^{2} \beta^{\prime}(n-m)\right)^{\beta_{0}} \varepsilon_{n} \\
& \leqq\left(1+\beta_{0}\right)^{2}(n-m)^{\beta_{0}} \varepsilon_{n},
\end{aligned}
$$

$$
\begin{aligned}
& R_{n} \leqq L R_{m}, \\
& R_{m} \leqq L\left(1+g_{n}^{2} \beta^{\prime}(n-m)\right)^{\beta_{0}} R_{n} \leqq(L+1)(n-m)^{\beta_{0}} R_{n} .
\end{aligned}
$$

Similar inequalities hold for other constants, which will be introduced later.

The domains $\Omega_{j}, \Lambda_{j}$, which are determined by he $j$-th renormalization transformation, but not by the $\mathbf{R}$-operation, are unions of $M R_{j}$-cubes in the lattice $T_{L^{-}}$. They satisfy also other conditions, e.g., the distance between their boundaries is at least equal to $2 M R_{j}$, which will be determined inductively by the operations in the $k+1$-st step.

In the first step the integration with respect to the variables $\left.U\right|_{\Omega_{1}^{c}}=V_{0}$ was left unchanged. Similarly in the next steps there are integrations with respect to variables on large field regions left. In $j$-th step the region is $\Omega_{j}^{c}$, and gauge field variables $V_{j-1}$ are defined on bonds of $\Omega_{j}^{(j-1) c}$. Obviously $\Gamma_{j-1} \subset \Omega_{j}^{(j-1) c}$, and we assume that the field $V_{j-1}$ is regular on $\Gamma_{j-1}$ in the sense that $\left|\partial V_{j-1}-1\right|$ $<O\left(L^{2}\right) \varepsilon_{j-1}$. The fields $V_{j}$ determine the field $V$ defined on $\mathbf{B}$ :

$$
V=V_{j} \quad \text { on } \Gamma_{j}, \quad j=0,1, \ldots, k .
$$

It is convenient also to introduce the averaging operation $M_{\mathbf{B}}$ associated with a determining set $\mathbf{B}$. It transforms a gauge field $U$ on the lattice $T_{\eta}$ into the gauge field $M_{\mathbf{B}}(U)$ defined on the set $\mathbf{B}$ by the equalities

$$
M_{\mathbf{B}}(U)=M^{j}(U) \quad \text { on } \Gamma_{j}, \quad j=0,1, \ldots, k .
$$

A regular configuration $V$ on $\mathbf{B}$ determines the minimal orbit, i.e., the set of minima, of the functional

$$
U \rightarrow A^{\eta}(U) \text { on } U: U \text { regular and } M_{\mathbf{B}}(U)=V .
$$

For precise definitions and the theory of this variational problem see [15]. A minimal configuration, i.e., an element of the unique minimal orbit, is denoted by $U_{\mathbf{B}}(V)$, or $U_{k}(V)$, or simply $U_{k}$, if there is no misunderstanding about the determining set $\mathbf{B}$, or the configuration $V$. These configurations, and their properties, provide crucial technical tools in our method, and we will use all the results of [15].

In constructions of this and subsequent sections and papers we will have to localize gauge field configurations, especially solutions of the variational problems, so it is convenient to describe some standard constructions, and to introduce some new notations. Consider a domain $\Omega$ such that it is a union of $M_{1}$-cubes in the lattice $T_{\xi}, \xi=L^{-j}$. For this domain we build a minimal determining set with a support in $\Omega$, or a sequence of maximal domains $\Omega=\Omega_{0} \supset \Omega_{1} \supset \ldots \supset \Omega_{j}$ such that $\Omega_{n}$ is a union of $L^{n} \xi M_{1}$-cubes, and $\operatorname{dist}\left(\Omega_{n}, \Omega_{n-1}^{c}\right) \geqq L^{n} \xi M_{1}, n=1, \ldots, j$ (the distance is for the lattice $\left.T_{\xi}\right)$. It is easy to see that $\operatorname{dist}\left(\Omega_{j}, \Omega^{c}\right) \leqq 2 M_{1}$, or $\Omega^{\sim-2} \subset \Omega_{j}$, where the operation $\sim$ is taken for $M_{1}$-cubes. We denote this determining set by $\mathbf{B}_{j}(\Omega)$, and the 
corresponding minimal configurations by

$$
U\left(\mathbf{B}_{j}(\Omega), \cdot\right)=U_{j, \Omega}(\cdot) .
$$

Later we will introduce simplified notations for some special cases. If we are given a determining set $\mathbf{B}$, and the corresponding function $U_{\mathbf{B}}$, then it is frequently necessary to localize them to a domain $\Omega$. Assume that $\partial \Omega \subset \Omega_{j} \backslash \Omega_{j+1}$, that $\Omega$ is a union of $M_{1}$-cubes in the lattice $T_{\xi}$, and that the distance between $\partial \Omega$ and this boundary of $\Omega_{j}, \Omega_{j+1}$, which is contained in $\Omega$, is greater than, or equal to $2 M_{1}$. Take the set $\mathbf{B}_{j}(\Omega)$, and form a new determining set by

$$
\mathbf{B} \cup \mathbf{B}_{j}(\Omega)=\left(\mathbf{B} \cap \Omega^{\sim-2}\right) \cup\left(\mathbf{B}_{j}(\Omega) \cap\left(\Omega \backslash \Omega^{\sim-2}\right)\right) .
$$

The corresponding function is denoted by

$$
U\left(\mathbf{B} \cup \mathbf{B}_{j}(\Omega), \cdot\right)=U_{\mathbf{B}, \Omega}(\cdot) .
$$

We can also have a more complicated situation, in which the boundary $\partial \Omega$ is contained in several different domains $\Omega_{j} \backslash \Omega_{j+1}$. Then we construct the corresponding determining sets restricted to the domains, and we define the set $\mathbf{B} \cup \mathbf{B}(\Omega)$ by (2.14), with the right-hand side summed up over the domains. The function determined by this set is denoted again by (2.15).

We consider the partition of the lattice $T_{\eta}$ into $L M_{2} R_{k}$-cubes, compatible with the other partitions, and for each cube $\square$ of this partition we define the function $U_{k, \square}\left(V_{k}\right)$ by

$$
U_{k, \square}\left(V_{k}\right)=U\left(\mathbf{B}_{k}\left(\square^{\sim 4}\right), M^{*}\left(Q_{k}^{s^{*}} V_{k}\right)\right),
$$

where $Q_{k}^{s^{*}} V_{k}$ is defined as in (1.3), only 1 -blocks are replaced by $k$-blocks. The notation in (2.16) is a slightly different, simplified version of the notation in (2.13).

Let us write now a general form of the expansion of the density $\varrho_{k}$. At first we define

$$
\chi_{k}\left(\Omega_{k}\right)=\prod_{\square \subset \Omega_{k}} \chi\left(\left\{\sup _{p \subset \square \sim}\left|U_{k, \square}\left(V_{k}, \partial p\right)-1\right|<\varepsilon_{k} \eta^{2}\right\}\right),
$$

where the cubes $\square$ belong to the partition of the lattice $T_{\eta}$ into cubes of the size $L M_{2} R_{k}$. This partition is compatible with all other partitions of this lattice. The density $\varrho_{k}\left(V_{k}\right)$ can be represented as

$$
\varrho_{k}\left(V_{k}\right)=\sum_{\left\{\Omega_{j}\right\},\left\{\Lambda_{j}\right\}} \chi_{k}\left(\Omega_{k}\right) \mathbf{T}_{k}\left(\left\{\Omega_{j}\right\},\left\{\Lambda_{j}\right\}\right) \exp A_{k}\left(\frac{1}{g_{k}^{2}}, U_{k}\right),
$$

where the summation is over the admissible sequences of domains. Summation over the sequences $\left\{S_{j}\right\}$ is included in the operation $\mathbf{T}_{k}$, and the effective action $A_{k}$ depends on the sequences $\left\{\Omega_{j}\right\},\left\{\Lambda_{j}\right\},\left\{S_{j}\right\}$. We suppress this dependence in our notation, so we will write simply $\mathbf{T}_{k}, A_{k}$.

At first we describe in general the operation $\mathbf{T}_{k}$. It was described in detail in the first step, and the complete inductive definition will follow from constructions of the subsequent sections. Basically this operation is a composition of integrations restricted to large field regions in successive scales, and multiplications by characteristic functions, $\delta$-functions defining renormalization transformations, 
and gauge fixing expressions. We start the general description with factorization properties. The last large field region is $Z_{k}$, and $\mathbf{T}_{k}$ is supported in it, in the sense that it involves integrations and variables restricted to this region. If $Z_{k}$ is represented as a union of disjoint regions, e.g. as a union of connected components, $Z_{k}=X_{1} \cup \ldots \cup X_{n}, X_{i} \cap X_{j}=\emptyset$ for $i \neq j$, then

$$
\mathbf{T}_{k}\left(Z_{k}\right)=\prod_{i=1}^{n} \mathbf{T}_{k}\left(X_{i}\right)
$$

The operations corresponding to disjoint regions commute, i.e., $\mathbf{T}_{k}\left(X_{i}\right) \mathbf{T}_{k}\left(X_{j}\right)$ $=\mathbf{T}_{k}\left(X_{j}\right) \mathbf{T}_{k}\left(X_{i}\right)$. For a given large field region $X$ the operation $\mathbf{T}_{k}(X)$ can be factorized into a product of one-step operations, and has the form

$$
\mathbf{T}_{k}(X)=\prod_{j=k-1}^{o} \mathbf{T}^{(j)}\left(Z_{j+1} \cap X\right)
$$

This is an ordered product, the order indicated in the product symbol. The operation $\mathbf{T}^{(j)}$ involves integration with respect to the gauge field variables $V_{j}$ on $\Omega_{j+1}^{c} \cap X$, and with respect to the fluctuation field variables $A_{j}$ on $Z_{j+1} \cap \Omega_{j+1} \cap X$, if the last set is nonempty. This operation comes from the renormalization transformation $T$ in the $j+1$-st step, and if it is not changed by an $\mathbf{R}$-operation, then it has the form

$$
\begin{aligned}
\mathbf{T}^{(j)}\left(Z_{j+1} \cap X\right)= & \left.\int d V_{j}\right|_{\Omega_{j+1}^{c} \cap X} \delta\left(\bar{V}_{j} V_{j+1}^{-1}\right) \zeta\left(\Omega_{j+1}^{c}\right) \\
& \left.\cdot \int d A_{j}\right|_{Z_{j+1} \cap \Omega_{j+1} \cap X} \chi\left(Z_{j+1} \cap \Omega_{j+1} \cap X\right) \exp \left[-\frac{1}{2}\left\langle A_{j}, C^{*} \Delta^{(j)} C A_{j}\right\rangle\right. \\
& \left.+\frac{1}{2}\left\langle A_{j}, C^{*} \Delta^{(j)} C C^{(j)}\left(\Lambda_{j+1}\right) C^{*} \Delta^{(j)} C A_{j}\right\rangle\right]
\end{aligned}
$$

More precisely, the quadratic form in the exponential couples only the fields $A_{j}$ in the same component of $Z_{j+1} \cap \Omega_{j+1}$. The other terms of this quadratic form are included into the effective action into $\mathbf{B}$-terms. If the operation $\mathbf{T}^{(j)}$ is changed by an R-operation, then the general form (2.21) is preserved, but the characteristic functions are changed, and the domain $Z_{j+1} \cap \Omega_{j+1} \cap X$ may be empty. We will use the factorization $(2.20)$ in the form

$$
\mathbf{T}_{k}(X)=\prod_{j=k-1}^{m} \mathbf{T}^{(j)}\left(Z_{j+1} \cap X\right) \mathbf{T}_{m}\left(Z_{m} \cap X\right),
$$

and in the case where the last $k-m$ operations have the form (2.21).

Now we describe the effective actions $A_{k}$. This is the most important part of the inductive assumption. The action $A_{k}$ is determined by the three sequences of sets, and it depends on the gauge field variable $V$, given by (2.10), through the background field $U_{k}(V)$. This field is determined by the sequence $\left\{\Omega_{j}\right\}$, or rather its determining set $\mathbf{B}$. Let us denote the system of the fluctuation fields $\left\{A_{j-1}\right\}$ by $A$. The effective action $A_{k}$ has the following general form:

$$
A_{k}\left(\frac{1}{g_{k}^{2}}, U_{k}\right)=-A\left(\frac{1}{g_{k}^{2}}, U_{k}\right)+\mathbf{E}_{k}\left(U_{k}\right)+\mathbf{R}_{k}\left(U_{k}\right)+\mathbf{B}_{k}\left(U_{k}, A\right)-E_{k} .
$$


All the expressions above depend on the sequences $\left\{\Omega_{j}\right\},\left\{\Lambda_{j}\right\}$, the expression $\mathbf{B}_{k}$ depends also on the sequence $\left\{S_{j}\right\}$. Before we describe them in detail let us explain briefly their meaining. The term $\mathbf{E}_{k}$ is the regular part of the action. It has the same properties as the effective action constructed in [I] for the small field approximation, but localized properly in different scales to domains determined by $\left\{\Lambda_{j}\right\}$. This expression is fully renormalized, i.e. vacuum energy and coupling constant renormalization counterterms are included into it. The term $\mathbf{R}_{k}$ arises as the effect of the $\mathbf{R}$-operations, and has localization properties similar to those of the term $\mathbf{E}_{k}$. For this term we perform the vacuum energy renormalization only. The term $\mathbf{B}_{k}$ includes various expressions localized closely to large field regions. It does not require any renormalization.

We start the detailed description of the terms in the effective action (2.23) with the definition of the function $g_{k}^{2}(x)$. It is defined by the sequence of the renormalization group equations generalzing Eqs. (I.0.20) and (1.27):

$$
\frac{1}{g_{j-1}^{2}(x)}=\frac{1}{g_{j}^{2}(x)}+\beta_{j}\left(g_{j-1}\right) \phi_{j}(x) \text {. }
$$

Here the coupling constants $g_{j-1}$ are defined as in (I.0.20), and $\phi_{j} \in C_{0}^{\infty}\left(\Lambda_{j}\right), \phi_{j}=1$ on $\Lambda_{j}^{-1}$. Thus $g_{j}^{2}(x)=g_{j}^{2}$ on the last domain.

The term $\mathbf{E}_{k}$ has the representation (I.1.7), but localized properly:

$$
\mathbf{E}_{k}\left(U_{k}\right)=\sum_{j=1}^{k}\left[\mathbf{E}^{(j)}\left(\Lambda_{j}, g_{j-1}, U_{k}\right)-\mathbf{E}^{(j)}\left(\Lambda_{j}, g_{j-1}, 1\right)-\beta_{j}\left(g_{j-1}\right) A\left(\phi_{j}, U_{k}\right)\right] .
$$

Localization properties of $\mathbf{E}^{(j)}$ are crucial for our analysis. We need a different, more detailed description than the one in (I.1.27), because now we must obtain more information about bounds for (2.25), and for localized expressions. Let us denote by $\Lambda_{j}^{0}$ the set which is obtained by removing one layer of the $M R_{j}$-cubes from $\Lambda_{j}^{(j)} \subset T_{1}^{(j)}$. The following representations hold:

$$
\begin{aligned}
& \mathbf{E}^{(j)}\left(\Lambda_{j}, U_{k}\right)=\sum_{z \in \Lambda_{j}^{0}} \mathbf{E}^{(j)}\left(\Lambda_{j}, U_{k}, z\right), \\
& \mathbf{E}^{(j)}\left(\Lambda_{j}, U_{k}, z\right)=\sum_{X \ni z} \mathbf{E}^{(j)}\left(X, U_{k}, z\right),
\end{aligned}
$$

where the last sum is over localization domains $X \in \mathbf{D}_{j}$ contained in $\Lambda_{j}, X \subset \Lambda_{j}$. The term $\mathbf{E}^{(j)}\left(X, U_{k}, z\right)$ of the last sum has the following properties: (i) it depends on $U_{k}$ restricted to $X$; (ii) there exists an analytic function $\mathbf{E}^{(j)}(X,(\mathbf{U}, \mathbf{J}), z)$ of the variables $(\mathbf{U}, \mathbf{J}) \in U_{j}^{c}\left(X, \alpha_{0, j}, \alpha_{1, j}\right)$, which is an extension of this term, i.e., the equality (I.1.9) is satisfied; (iii) the extended function is invariant with respect to the gauge transformations (I.1.10); (iv) it satisfies the inequality (I.1.18). These properties are the same as in [I.1]. The numbers $\alpha_{0, j}, \alpha_{1, j}$ in the symbol of the space are given by

$$
\alpha_{0, j}=g_{j} C_{0}\left(\log g_{j}^{-2}\right)^{q_{0}}, \quad \alpha_{1, j}=g_{j} C_{1}\left(\log g_{j}^{-2}\right)^{q_{1}},
$$

where $q_{0}, q_{1}$ are integers greater than $1, C_{0}, C_{1}$ are sufficiently large positive numbers. 
The last important property is the Euclidean covariance. We have it only for functions $\mathbf{E}^{(j)}\left(U_{j}, z\right)$ defined by the equality (2.27) with the unrestricted summation, i.e., with the sum over all $X \in \mathbf{D}_{j}$ containing the point $z$. These functions satisfy the equality

$$
\mathbf{E}^{(j)}\left(r U_{j}, r z\right)=\mathbf{E}^{(j)}\left(U_{j}, z\right)
$$

for arbitrary Euclidean transformations $r$ of the lattice $T^{(j)}$, and for regular configurations $U_{j}$. This implies that the function $\mathbf{E}^{(j)}\left(U_{j}, z\right)$ is invariant with respect to the Euclidean transformations of $U_{j}$ leaving the point $z$ invariant.

The term $R_{k}$ has the renormalization similar to (2.25):

$$
\mathbf{R}_{k}\left(U_{k}\right)=\sum_{j=1}^{k}\left[\mathbf{R}^{(j)}\left(\Lambda_{j}, U_{k}\right)-\mathbf{R}^{(j)}\left(\Lambda_{j}, 1\right)\right] .
$$

Each function $\mathbf{R}^{(j)}\left(\Lambda_{j}, U_{k}\right)$ has the localized renormalization of the form (I.1.7), but with the summation over localization domains $X \in \mathbf{D}_{j}$ contained in $\Lambda_{j}^{\sim-1}, X \subset \Lambda_{j}^{\sim-1}$, which are unions of the $M R_{j}$-cubes in the lattice $T_{\xi}$. Terms of this representation have the properties (i)-(iii) formulated above, after (2.27). The property (iv) is different now; we assume that the following stronger inequality holds :

$$
\left|\mathbf{R}^{(j)}(X,(\mathbf{U}, \mathbf{J}))\right| \leqq g_{j}^{\kappa_{0}} \exp \left(-\kappa d_{j}(X)\right)
$$

Here $\kappa_{0}$ can be chosen arbitrarily large, similarly as $\kappa$, if the other parameters are fixed properly, as in [I]. The above inequality is connected with the fact that the terms are defined by integrals including large field domains of integrations. It explains why coupling constant renormalization counterterms are not needed in (2.30). After the vacuum energy renormalization we obtain a sum of marginal terms, i.e., terms with bounds $O(1)\left(L^{j} \eta\right)^{4} g_{j}^{\kappa 0} \exp \left(-\kappa d_{j}(X)\right)$. The sum over $X$ is controlled by the exponential factor, and by the factor $\left(L^{j} \eta\right)^{4}$. The sum over $j$ is controlled by $g_{j}^{\kappa_{0}}$.

The functions $\mathbf{R}^{(j)}\left(X, U_{j}\right)$ have the Euclidean covariance property

$$
\mathbf{R}^{(j)}\left(r X, r U_{j}\right)=\mathbf{R}^{(j)}\left(X, U_{j}\right)
$$

holding for these Euclidean transformations $r$, which leave the partition of the lattice $T_{\xi}$ in $M R_{j}$-cubes invariant. The above property will follow immediately from the construction of the $\mathbf{R}$-terms given in the next paper. If we sum up all these terms over all the admissible domains $X$ in $T_{\xi}$, we get a Euclidean invariant function $\mathbf{R}^{(j)}\left(U_{j}\right)$, i.e.,

$$
\mathbf{R}^{(j)}\left(r U_{j}\right)=\mathbf{R}^{(j)}\left(U_{j}\right), \quad \text { where } \quad \mathbf{R}^{(j)}\left(U_{j}\right)=\sum_{X} \mathbf{R}^{(j)}\left(X, U_{j}\right),
$$

and the transformations $r$ are as above.

The term $\mathbf{B}_{k}$ is the simplest term from the point of view of the renormalization, it does not need any renormalization, but it has the most complicated structure and properties. It is a sum of small, or at least bounded, localized terms, with localizations close to large field regions.

The terms of $\mathbf{B}_{k}$ are analytic functions of the variables $(\mathbf{U}, \mathbf{J})$ introduced in the same way as in the regular cases before. The analyticity domains are spaces defined 
similarly as in (I.1.11)-(I.1.16), but now we must take into account the existence of many different scales. We introduce the following definition.

The space $\widetilde{U}_{j}^{c}\left(X, \tilde{\alpha}_{0}, \tilde{\alpha}_{1}\right)$ for $X \in \mathbf{D}_{j}$ is the set of configurations $(\mathbf{U}, \mathbf{J})$ defined on $X$ and satisfying the three conditions below.

(i) $\mathbf{U}=U^{\prime} U, U$ has values in the group $G$, and the configurations

$$
U, \mathbf{U}, U_{p, X}\left(M^{*}(\mathbf{U})\right)=U\left(\mathbf{B}_{p}(X) \cup\left\{\Gamma_{i}\right\}_{i<p}, M^{*}(\mathbf{U})\right), \mathbf{J}, J_{p, X}\left(M^{*}(\mathbf{U})\right)
$$

satisfy the bounds

$$
\begin{gathered}
|\partial U-1|,|\partial \mathbf{U}-1|<\left(1-\beta\left(1-2^{-(j-n)}\right)\right) \alpha_{0, n} \xi^{2}\left(L^{n} \xi\right)^{-2}, \\
\left|\partial U_{p, X}\left(M^{\cdot}(\mathbf{U})\right)-1\right|<\left(1-\beta\left(1-2^{-(j-n)}\right)\right) \alpha_{0, n} L^{-2 p}\left(L^{n} L^{-p}\right)^{-2}, \\
|\mathbf{J}|<\left(1-\beta\left(1-2^{-(j-n)}\right)\right) \alpha_{0, n}\left(L^{n} \xi\right)^{-3}, \\
\left|\mathbf{J}_{p, X}\left(M^{\cdot}(\mathbf{U})\right)\right|<\left(1-\beta\left(1-2^{-(j-n)}\right)\right) \alpha_{0, n} L^{n-\min \{p, n\}}\left(L^{n} L^{-p}\right)^{-3},
\end{gathered}
$$

on $X \cap\left(\Omega_{n} \backslash \Omega_{n+1}\right)$ for $n=1, \ldots j-1$, or on $X \cap \Omega_{j}$ for $n=j$.

(ii) For each cube $\square \subset \Omega_{n} \backslash \Omega_{n+2}, \square \cap \Omega_{n+1}^{c} \neq \emptyset, n=1, \ldots, j-1$, of the size $C M L^{n} \xi$, or $\square \subset \Omega_{j}$ and of the size $C M$, there exists a gauge transformation $u$ defined on $\square \cap X$ and such, that

$$
U^{u}=\exp i \xi A, L^{n} \xi|A|,\left(L^{n} \xi\right)^{2}\left|\nabla^{\xi} A\right|<B C M \alpha_{0, n}
$$

on $\square \cap X$, with an absolute constant $B$.

(iii) $U^{\prime}=\exp i \xi A^{\prime}, A^{\prime}$ has values in the algebra $\mathbf{g}^{\mathrm{c}}$,

$$
L^{n} \xi\left|A^{\prime}\right|,\left(L^{n} \xi\right)^{2}\left|\nabla_{U}^{\xi} A^{\prime}\right|<\left(1-\beta\left(1-2^{-(j-n)}\right)\right) \alpha_{1, n}
$$

on $X \cap\left(\Omega_{n} \backslash \Omega_{n+1}\right)$ for $n=1, \ldots, j-1$, or on $X \cap \Omega_{j}$ for $n=j$.

The number $\beta$ is a small positive constant, but not too small, e.g., we can take $\beta=1 / 4$. The spaces defined above are invariant with respect to $G$-valued gauge transformations. This definition gives a more detailed and precise form, suitable for our inductive constructions, of the regularity conditions for background fields, introduced in [13-15].

Now we can write the form of $\mathbf{B}_{k}$. It is given by the sum

$$
\sum_{j=1}^{k} \mathbf{B}^{(j)}\left(U_{k}, A,\left\{S_{i}\right\}\right),
$$

and a term in this sum has the localized representation

$$
\mathbf{B}^{(j)}\left(U_{k}, A,\left\{S_{i}\right\}\right)=\sum_{X} \mathbf{B}^{(j)}\left(X, U_{k}, A,\left\{S_{i} \cap X\right\}\right),
$$

where the sum is over domains $X \in \mathbf{D}_{j}$ such, that $X \cap \Omega_{j} \neq \emptyset$, and $X \cap Z_{j}^{\sim} \neq \emptyset$. The localized term in the sum on the right-hand side above has the following properties: (i) it depends on $U_{k}, A$ restricted to $X$; (ii) it has an extension to an analytic function on the space $\tilde{U}_{j}^{c}\left(X, \tilde{\alpha}_{0}, \tilde{\alpha}_{1}\right)$; (iii) the extended function is invariant with respect to ( $G$-valued) gauge transformations of $\mathbf{U}, \mathbf{J}, A$ (the variables $\mathbf{J}, A$ are transformed by the adjoint representation of the gauge transformations); (iv) it satisfies the bound

$$
\left|\mathbf{B}^{(j)}\left(X,(\mathbf{U}, \mathbf{J}), A,\left\{S_{i} \cap X\right\}\right)\right|<B_{0} \exp \left(-\kappa d_{j}(X)\right) .
$$


The terms have a bit more precise localization property. Denote $\Omega_{0}=\Omega_{1}^{\sim}$, and consider $X \cap \Omega_{0}$. It is a union of components. If a component does not connect to $\Omega_{j}$, i.e., if the intersection of this component with $\Omega_{j}$ is empty, then the functions does not depend on the fields $(\mathbf{U}, \mathbf{J}), A$ restricted to this component.

There are other possible forms of the inductive assumptions for the boundary terms. For example, we may resum all the terms with localization domains intersecting a given component of the large field region. This gives sum (2.41), with the summation over domains $X \in \mathbf{D}_{j}$ such that $X$ either contains a component of the large field region $Z_{j}$, or is disjoint with it. Resumming we lose a part of the exponential factor in (2.42), connected with the region $Z_{j}$; hence for the new terms we obtain the bound (2.42) with $d_{j}(X)$ replaced by $d_{j}\left(X \backslash Z_{j}\right)$. All constructions and proofs of the procedure can be carried on with these inductive assumptions. We have chosen the ones above, because they agree with the assumptions for E-terms and R-terms.

The constant $E_{k}$ (depending on $\left\{\Omega_{j}\right\},\left\{\Lambda_{j}\right\}$ also) is obtained by subtracting onestep vacuum energy expressions, generated in small field regions, from the initial constant $E$. This initial constant is defined in fact as a sum of all such expressions for all the lattices $T^{(k)}$ as the small field regions. The constant $E_{1}$ was defined in Sect. 1, and a general inductive definition will be given in the next section.

All the inductive assumptions are formulated for the effective density obtained after the $k$-th operation $\mathbf{R} T$. It is a composition of the operations $T$ and $\mathbf{R}$, applied in this order, and after the operation $T$ we obtain expressions with better analyticity and decay properties. More precisely, the expressions with indices $j<k$ are exactly as described above, but the newly created expressions $\mathbf{E}^{(k)}, \mathbf{R}^{(k)}, \mathbf{B}^{(k)}$ are defined on slightly larger spaces, with the coefficients in their definition bigger by $\beta$ multiplied by a corresponding number, and they have better decay properties, with the number $\kappa$ replaced, for example, by $(1+4 \beta) \kappa$. Such improved bounds are needed for the R-operation.

Now we are ready to formulate the basic results of this paper. The first theorem is a generalization of Theorem 3 [I].

Theorem 1. There exist constants, introduced in the above description, such that if the sequence of coupling constants $\left\{g_{k}\right\}$, determined by the recursive renormalization group (Callan-Symanzik) equations (0.18), (0.20) [I], satisfies the inequalities (I.0.33), then the sequence of densities $\left\{\varrho_{k}\right\}$, generated by successive applications of the operations $\mathbf{R} T$ to the density $\varrho_{0}=\exp \left[-\left(1 / g_{0}^{2}\right) A-E\right]$, satisfies all the inductive assumptions. The constants satisfy numerous restrictions introduced in the proof.

Let us make two remarks connected with the formulation of the above theorem. At first, some of the restrictions on constants, e.g. on constants $\kappa, M, \varepsilon_{j}, \alpha_{0, j}, \alpha_{1, j}$, come from the paper [I]. We assume that all of them are satisfied here, and we will introduce some new restrictions also.

The second remark concerns a possible generalization of this theorem. For a given index $k$ we introduce the space of all densities satisfying the conditions of the inductive assumption. The theorem states that the operation $\mathbf{R} T$ transforms the space with the index $k$ into the space with the index $k+1$. This generalization does not seem to be useful, or interesting now. 
Obviously we are interested not only in getting representations of the effective densities, but also in proving uniform bounds for them. We state now several results of this type. We formulate bounds for the effectiove actions separately. We start with localized expressions and bounds. Let $\Omega$ be a domain from $\mathbf{D}_{k}$ contained in $B^{j}\left(\Lambda_{j}^{0}\right)$, and let $\phi \in C_{0}^{\infty}\left(\Omega^{\sim}\right), \phi=1$ on $\Omega$. Notice that here $\Omega^{\sim}$ denotes the union of the domains $\left(\Omega \cap\left(\Omega_{n} \backslash \Omega_{n+1}\right)\right)^{\sim}, n=j, \ldots, k$, the operation $\sim$ is in the corresponding scale.

Theorem 2. Under the assumptions of Theorem 1 there exists a constant $E_{1}$ independent of $j, k, \Omega,\left\{\Omega_{j}\right\},\left\{\Lambda_{j}\right\}, T$ (but dependent on the other constants occurring in the formulation of this theorem), such that

$$
\begin{aligned}
& \left|\sum_{z \in \Lambda_{j}^{0} \cap \Omega}\left[\mathbf{E}^{(j)}\left(\Lambda_{j}, U_{k}, z\right)-\mathbf{E}^{(j)}\left(\Lambda_{j}, 1, z\right)\right]-\beta_{j}\left(g_{j-1}\right) A\left(\phi, U_{k}\right)\right| \\
& \quad \leqq E_{1} \sum_{n=j}^{k}\left(L^{j-n}\right)^{\beta}\left|\Gamma_{n} \cap \Omega\right|,
\end{aligned}
$$

for $\beta<1$, and sufficiently regular configurations $U_{k}=U_{k}(V)$, e.g. for $V$ restricted by the characteristic functions in (2.18). The constant $E_{1}$ depends on $\beta$ also, and grows to $\infty$ if $\beta \rightarrow 1$. The volumes are taken in the corresponding scales, i.e. $\left|\Gamma_{n} \cap \Omega\right|$ means the number of points in the set $\Gamma_{n} \cap \Omega \subset T_{1}^{(n)}$. Similarly, there exists an absolute constant $R_{1}$ such, that

$$
\left|\sum_{X \in \mathbf{D}_{j}, X \subset \Lambda_{J}, X \cap \Omega \neq \emptyset}\left[\mathbf{R}^{(j)}\left(X, U_{k}\right)-\mathbf{R}^{(j)}(X, 1)\right]\right| \leqq R_{1} g_{j}^{\kappa_{0}} \sum_{n=j}^{k}\left|\Gamma_{n} \cap \Omega\right|,
$$

for the regular configurations $U_{k}$. (In fact the constant $R_{1}$ can be taken as equal to 1 for $g_{j}$ sufficiently small.)

The most essential part of this theorem is the inequality (2.43). It is an immediate consequence of the inequality with the set $\Lambda_{j}^{0} \cap \Omega$ replaced by one point, and with the function $\phi$ replaced by a function, which is localized in a neighborhood of this point, and which is an element of a special decomposition of unity. The new, more detailed representation (2.26), (2.27) of this part of the effective action is introduced in order to prove this inequality. The second inequality $(2.44)$ is more elementary and easier to prove. It follows from the considerations of Sects. 3, 4 [I]. Let us write now some consequences of these inequalities. Taking $\Omega=B^{j}\left(\Lambda_{j}^{0}\right), \phi=\phi_{j}$ in (2.43), and summing the obtained inequalities over $j$, we get

$$
\left|\mathbf{E}_{k}\left(U_{k}\right)\right| \leqq E_{1} \sum_{n=1}^{k}\left|\Gamma_{n}\right| \sum_{j=1}^{n}\left(L^{j-n}\right)^{\beta}<E_{1}\left(1-L^{-\beta}\right)^{-1} \sum_{n=1}^{k}\left|\Gamma_{n}\right| .
$$

Similarly, taking $\Omega=B^{j}\left(\Lambda_{j}^{0}\right)$ in (2.44), and summing over $j$, we get

$$
\left|\mathbf{R}_{k}\left(U_{k}\right)\right| \leqq R_{1} \sum_{n=1}^{k}\left|\Gamma_{n}\right| \sum_{j=1}^{n} g_{j}^{\kappa_{0}}<R_{1} \sum_{n=1}^{k}\left|\Gamma_{n}\right| g_{n}^{\kappa_{0}-6}<\sum_{n=1}^{k}\left|\Gamma_{n}\right|,
$$

for $\kappa_{0} \geqq 7$ and $g$ sufficiently small. Bounds for the boundary terms (2.40) are even more elementary. We use the exponential factor $\exp \left(-(\kappa-1) d_{j}(X)\right)$ from the inequality (2.42) to control the sum over $X$ in (2.41). The remaining factor 
$\exp \left(-d_{j}(X)\right)$ is used to get an additional small factor, e. g. for $j>n$ it can be bounded by $2^{-(j-1-n)}$. Thus we obtain

$$
\left|\mathbf{B}^{(j, n)}\left(U_{k}, A,\left\{S_{i}\right\}\right)\right| \leqq B_{1} 2^{-(j-n)}\left|\Gamma_{n}\right|
$$

for $j>n$, and this inequality with $\left|\Gamma_{n} \backslash \Lambda_{k}^{0}\right|$ for $j=n$. Summing over $j$ from $n$ to $k$, and then over $n$ from 1 to $k$, we get

$$
|(2.40)| \leqq 2 B_{1}\left(\sum_{n=1}^{k-1}\left|\Gamma_{n}\right|+\left|\Gamma_{k} \backslash \Lambda_{k}^{0}\right|\right) \leqq 2 B_{1} \sum_{n=1}^{k}\left|\Gamma_{n}\right| .
$$

Finally, the vacuum energy counterterm $E_{k}$, except the terms logarithmic in coupling constants, has the same bound as above, only with a different constant.

These bounds yield the following bounds for the effective actions

$$
A_{k}\left(\frac{1}{g_{k}^{2}}, U_{k}\right)=-A\left(\frac{1}{g_{k}^{2}}, U_{k}\right)+(\text { the logarithmic terms })+O(1) \sum_{j=1}^{k}\left|\Gamma_{j}\right| \text {. }
$$

The first term on the right-hand side yields the small factors for large field characteristic functions. It also controls the logarithmic terms. Thus we estimate the integral $\int d V_{k} \varrho_{k}$ by a sum of terms similar to the one considered in Sect. 3 [6], e.g., see (3.42). The procedure is constructed in such a way, that the combinatorics now is the same, relative to the $\eta$-scale of the lattice $T_{\eta}$, as in [6], hence we have the same result for this scale.

Corollary 3 (Ultraviolet Stability). Under the assumptions of Theorem 1 there exist constants $E_{-}, E_{+}$independent of $\eta$ and $T$, but depending on $g_{k}$, such that

$$
\chi_{k}\left(T_{\eta}\right) \exp \left[-\frac{1}{g_{k}^{2}} A\left(U_{k}\left(V_{k}\right)\right)-E_{-}\left|T_{\eta}\right|\right] \leqq \varrho_{k}\left(V_{k}\right) \leqq e^{E_{+}\left|T_{\eta}\right|} .
$$

\section{The $k+1$-st Renormalization Transformation and the Proof of Theorem 2}

We apply the next renormalization transformation to the density $\varrho_{k}$, and we study the generation of the new density, and especially the terms $\mathbf{E}^{(k+1)}, \mathbf{R}^{(k+1)}, \mathbf{B}^{(k+1)}$, $E^{(k+1)}$. The inductive assumptions and Theorem 2 are proved for these terms. Almost all operations and bounds were studied in the previous papers [16, I], so we concentrate only on new issues.

Using the inductive assumption we write

$$
\left(T \varrho_{k}\right)\left(V_{k+1}\right)=\sum_{\left\{\Omega_{J}\right\},\left\{\Lambda_{j}\right\}} \int d V_{k} \delta\left(\bar{V}_{k} V_{k+1}^{-1}\right) \chi_{k} \mathbf{T}_{k} \exp A_{k},
$$

and next we consider one term on the right-hand side. Notice that the restrictions ontroduced by the characteristic function $\chi_{k}$ imply that the new fields $V_{k+1}$ satisfy the bounds $\left|V_{k+1}\left(\partial p^{\prime}\right)-1\right|<2 L^{2} \varepsilon_{k}$ for $p^{\prime} \in \Gamma_{k}^{(1)}$. We introduce new restrictions on these fields. The term has a large field region $Z_{k}=\Lambda_{k}^{c}$, and we denote by $Z_{k}^{\prime}$ the union of all $L M R_{k+1}$-cubes intersecting the region $Z_{k}$, and belonging to the partition of the lattice $T_{\eta}$. This partition, or the corresponding partition of the lattice $T_{L^{-1} \eta}$ into $M R_{k+1}$-cubes, is compatible with all the previous partitions. We surround $Z_{k}^{\prime}$ by 
four layers of the $L M R_{k+1}$-cubes, i.e., we take the domain $Z_{k}^{\prime \sim 4}$, and we introduce the following decomposition of unity for the domain $\left(Z_{k}^{\prime \sim 4}\right)^{c}$ :

$$
\begin{aligned}
1= & \sum_{P_{k+1}} \prod_{\square^{\prime} \subset P_{k+1}^{c}} \chi\left(\left\{\sup _{p \subset \square^{\prime} \sim}\left|U_{k+1, \square^{\prime}}(\partial p)-1\right|<\varepsilon_{k+1}\left(L^{-1} \eta\right)^{2}\right\}\right) \\
& \cdot \prod_{\square^{\prime} \subset P_{k+1}} \chi\left(\left\{\sup _{p \subset \square^{\prime} \sim}\left|U_{k+1, \square^{\prime}}(\partial p)-1\right| \geqq \varepsilon_{k+1}\left(L^{-1} \eta\right)^{2}\right\}\right) \\
= & \sum_{P_{k+1}} \chi_{k+1}\left(P_{k+1}^{c}\right) \chi_{k+1}^{c}\left(P_{k+1}\right),
\end{aligned}
$$

where the summation is over domains $P_{k+1} \subset\left(Z_{k}^{\prime \sim 4}\right)^{c}$, which are unions of $L^{2} M_{2} R_{k+1}$-cubes. The cubes are from the partition of the lattice $T_{\eta}$ into cubes of the size $L^{2} M_{2} R_{k+1}$, or the partition of the lattice $T_{L^{-1} \eta}$ into cubes of the size $L M_{2} R_{k+1}$. The partition is compatible with all the other partitions. We take the set $P_{k+1}^{\prime}$ - the union of all $L M R_{k+1}$-cubes intersecting $P_{k+1}$ - and we surround the union $P_{k+1}^{\prime} \cup Z_{k}^{\prime \sim 4}$ by a layer of $L M R_{k+1}$-cubes. In the complement $\left(P_{k+1}^{\prime \sim} \cup Z_{k}^{\prime \sim 5}\right)^{c}$ we introduce the axial gauge fixing as in (1.5), with $V_{k}, \varepsilon_{k}, g_{k}$ instead of $U, \varepsilon_{0}, g_{0}$. Denote by $P_{k+1}^{1}$ the subset of $T^{(k+1)}$ such that $B^{k+1}\left(P_{k+1}^{1}\right)=\left(P_{k+1}^{\prime \sim}\right)^{c} \cap\left(Z_{k}^{\prime \sim 5}\right)^{c}$. We get an expansion of the form (1.6), with the corresponding changes. Next, we surround the domain $P_{k+1}^{\prime \sim} \cup Z_{k}^{\prime \sim 5}$ by a layer of $L M R_{k+1}$-cubes, or we take off such a layer from $B^{k+1}\left(P_{k+1}^{1}\right)$, and in the domain $\left(P_{k+1}^{\prime \sim 2} \cup Z_{k}^{\prime \sim 6}\right)^{c}$ $=\left(B^{k+1}\left(P_{k+1}^{1}\right)\right)^{\sim-1}$ we introduce the decomposition of unity restricting the approximate fluctuation fields,

$$
\begin{aligned}
1= & \sum_{Q_{k+1}} \prod_{\square^{\prime} c Q_{k+1}^{c}} \chi\left(\left\{\sup _{b \in\left(\square^{\prime} \sim 2\right)^{(k) *}}\left|V_{k}(b)\left(V_{\square^{\prime}}^{(k)}(b)\right)^{-1}-1\right|<2 \delta_{k}\right\}\right) \\
& \cdot \prod_{\square^{\prime}\left(Q_{k+1}\right.} \chi\left(\left\{\sup _{b \in\left(\square^{\prime} \sim 2\right)^{(k) *}}\left|V_{k}(b)\left(V_{\square^{\prime}}^{(k)}(b)\right)^{-1}-1\right| \geqq 2 \delta_{k}\right\}\right) \\
= & \sum_{Q_{k+1}} \chi_{k}^{\prime}\left(Q_{k+1}^{c}\right) \chi_{k}^{\prime c}\left(Q_{k+1}\right) .
\end{aligned}
$$

The summation is over subdomains $Q_{k+1} \subset\left(B^{k+1}\left(P_{k+1}^{1}\right)\right)^{\sim-1}$, which are unions of $L^{2} M_{2} R_{k+1}$-cubes, and the complement is relative to the domain $\left(B^{k+1}\left(P_{k+1}^{1}\right)\right)^{\sim-1}$. The configuration $V_{\square}^{(k)}$ is defined on $\left(\square^{\prime \sim}\right)^{(k)}$ by

$$
V_{\square^{\prime}}^{(k)}=M^{k}\left(U_{k+1, \square^{\prime}}\right),
$$

and $\delta_{k}=g_{k} A_{1} / A_{0} p_{0}\left(g_{k}\right)$. We denote again by $Q_{k+1}^{\prime}$ the union of $L M R_{k+1}$-cubes intersecting $Q_{k+1}$, and we surround the domain $Q_{k+1}^{\prime} \cup\left(\left(B^{k+1}\left(P_{k+1}^{1}\right)^{c}\right)^{\sim}\right.$ by two layers of such cubes. Denote

$$
\Omega_{k+1}=\left(Q_{k+1}^{\prime \sim 2} \cup\left(B^{k+1}\left(P_{k+1}^{1}\right)^{c}\right)^{\sim 3}\right)^{c},
$$

hence $\left(Q_{k+1}^{\sim 2}\right)^{c}=Q_{k+1}^{\prime} \cup\left(B^{k+1}\left(P_{k+1}^{1}\right)^{c}\right)^{\sim}$. On the domain $\Omega_{k+1}^{\sim 2}$ there are only the small field characteristic functions from the above decompositions of unity. Now we prove that on $\Omega_{k+1}^{\sim}$ the functions $\chi_{\mathrm{Ax}}$ and $\chi_{k}$ are equal to 1 . Take $\chi_{k}(\square)$ for a cube $\square \subset \Omega_{k+1}^{\sim}$. This cube determines a cube $\square^{\prime}$ of the next partition, such that 
$\square \subset \square^{\prime} \subset \Omega_{k+1}^{\sim}$. For the function $U_{k, \square}$ we have

$$
\begin{aligned}
U_{k, \square} & =U\left(\mathbf{B}_{k}\left(\square^{\sim 4}\right),\left[M^{\cdot}\left(Q_{k}^{s^{*}} V_{k}\right)\left(M^{\cdot}\left(U_{k+1, \square^{\prime}}\right)\right)^{-1}\right] M^{\cdot}\left(U_{k+1, \square^{\prime}}\right)\right) \\
& =\left(\exp i \eta \mathbf{H}_{k, \square}\left(\frac{1}{i} \log \left[M^{\cdot}\left(Q_{k}^{s^{*}} V_{k}\right)\left(M^{\cdot}\left(U_{k+1, \square^{\prime}}\right)\right)^{-1}\right]\right) U_{k+1, \square^{\prime}}\right)^{u_{k, \square}^{-1}} .
\end{aligned}
$$

On almost the whole cube $\square^{\sim 4}$, except a boundary layer of the width $2 M_{1}$, the field in the argument of the function $\mathbf{H}_{k, \square}$ is equal to $(1 / i) \log \left[V_{k}\left(V_{\square}^{(k)}\right)^{-1}\right]$, hence it can be bounded by $4 \delta_{k}$. On the boundary layer this field can be bounded by $30 d^{2} L^{2} B_{3}(1$ $\left.+\beta_{0}\right) \varepsilon_{k}$ by an application of the inequality (1.65) from [14], or rather by an application of the reasoning leading to that inequality. By the exponential decay property (190) [15] we obtain the estimate

$$
\begin{aligned}
\left|\mathbf{H}_{k, \square}\right|,\left|\nabla_{U_{k+1, \square}}^{\eta} \mathbf{H}_{k, \square}\right| \leqq & B_{3}\left(4 \delta_{k}+\exp \left(-\delta 2 M_{2} R_{k}\right) 30 d^{2} L^{2} B_{3}\left(1+\beta_{0}\right) \varepsilon_{k}\right) \\
& <\left(4 B_{3} \frac{A_{1}}{A_{0}}+30 d^{2} L^{2} B_{3}^{2}\left(1+\beta_{0}\right) \exp \left(-R_{k}\right)\right) \varepsilon_{k} \\
& <\frac{1}{10} \varepsilon_{k} \text { on } \square^{\sim},
\end{aligned}
$$

for $A_{1} / A_{0}$ and $\gamma$ sufficiently small. This estimate and the equalities (3.7), (1.43) [14] imply

$$
\begin{aligned}
\left|U_{k, \square}(\partial p)-1\right| & <\left(1+\frac{2}{10} \varepsilon_{k} \eta\right) \varepsilon_{k+1}\left(L^{-1} \eta\right)^{2}+\frac{2}{10} \varepsilon_{k} \eta^{2}\left(1+\frac{4}{10} \varepsilon_{k}\right) \\
& <2\left(1+\beta_{0}\right) L^{-2} \varepsilon_{k} \eta^{2}+\frac{4}{10} \varepsilon_{k} \eta^{2}<\varepsilon_{k} \eta^{2} \quad \text { for } \quad p \subset \square^{\sim}
\end{aligned}
$$

Thus $\chi_{k}(\square)=1$ for $\square \subset \Omega_{k+1}$. For a function from $\chi_{\mathrm{Ax}}$ we have a simpler situation. Using the axial gauge for $V_{\square}^{(k)}$, we have

$$
\begin{aligned}
\left|V_{k}(y, x)-1\right| & =\left|V_{k}(y, x)\left(V_{\square}^{(k)}(y, x)\right)^{-1}-1\right|<O(1) \delta_{k} \\
& =O(1) \frac{A_{1}}{A_{0}} \varepsilon_{k}<\varepsilon_{k} \text { for } y \in\left(\Omega_{k+1}^{\sim}\right)^{(k+1)}, \quad x \in B(y),
\end{aligned}
$$

and for $A_{1} / A_{0}$ sufficiently small. Thus $\chi_{\mathrm{Ax}}\left(\left(\Omega_{\bar{k}+1}\right)^{(k)}\right)=1$.

On the domain $\Omega_{k+1}$ we introduce a fluctuation field as in [16]. Define the field $V^{(k)}$ on $\Omega_{k+1}^{(k)}$ as the minimum of the functional

$$
\begin{gathered}
\left.V_{k}\right|_{\Omega_{k+1}^{(k)} \rightarrow A\left(U_{k}\right)} \text { for } V_{k}: \bar{V}_{k}=V_{k+1} \text { on } \Gamma_{k+1}=\Omega_{k+1}^{(k+1)}, \\
V_{k}(y, x)=1 \text { for } y \in \Gamma_{k+1}, \quad x \in B(y) .
\end{gathered}
$$

This minimum is given by $V^{(k)}=M^{k}\left(U_{k+1}\right)$, where the minimal configuration $U_{k+1}$ is determined by the sequence of domains $\left\{\Omega_{k+1}, \Omega_{k}, \ldots\right\}$, and the corresponding sequence of fields $\left\{\left.V_{k+1}\right|_{\Gamma_{k+1}},\left.V_{k}\right|_{\Gamma_{k}}, \ldots\right\}$. The fluctuation field $V_{k}^{\prime}$ is defined now by

$$
V_{k}^{\prime}=V_{k}\left(V^{(k)}\right)^{-1} \text { on } B\left(\Gamma_{k+1}\right) \text {. }
$$


Consider a cube $\square^{\prime} \subset \Omega_{k+1}$. The restrictions on the fields $V_{k}, V_{k+1}$ on $\Omega_{k+1}^{\sim}$, and Theorem 1 from [16] imply, that $U_{k+1}$ satisfies the following regularity condition:

$$
\left|U_{k+1}(\partial p)-1\right|<2 B_{3} L^{2}\left(1+\beta_{0}\right) \varepsilon_{k}\left(L^{-1} \eta\right)^{2} \quad \text { for } \quad p \subset \square^{\prime} \text {. }
$$

On this cube the configuration $U_{k+1, \square}$, satisfies the regularity condition as above, only with the coefficient 1 in front of $\varepsilon_{k+1}$. By the definition of these configurations, and the constraints on $V_{k}$, we have $M^{k+1}\left(U_{k+1}\right)=V_{k+1}=M^{k+1}\left(U_{k+1}, \square^{\prime}\right)$ on $\square^{\prime \sim}$. Applying Lemma 1 [14] we obtain

$$
\left|V^{(k)}-V_{\square^{\prime}}^{(k)}\right|<(4 d L)^{2}\left(1+\beta_{0}\right) B_{3} \varepsilon_{k} \quad \text { on } \quad \square^{\prime \sim}
$$

hence

$$
\begin{aligned}
\left|V_{k}^{\prime}-1\right| & =\left|V_{k}-V^{(k)}\right| \leqq\left|V_{k}-V_{\square^{\prime}}^{(k)}\right|+\left|V_{\square^{\prime}}^{(k)}-V^{(k)}\right| \\
& <2 \delta_{k}+(4 d L)^{2}\left(1+\beta_{0}\right) B_{3} \varepsilon_{k}=O(1) \varepsilon_{k} \text { on } \square^{\prime}
\end{aligned}
$$

This implies that $\left|V_{k}^{\prime}-1\right|<O(1) \varepsilon_{k}$ on $\Omega_{k+1}$, because $\square^{\prime}$ is an arbitrary cube contained in $\Omega_{k+1}$. We define $A_{k}^{\prime}=(1 / i) \log V_{k}^{\prime}$, hence $\left|A_{k}^{\prime}\right|<O(1) \varepsilon_{k}$ also.

This way each term in (3.1) has been written as a sum of terms of the same type, but with the new characteristic functions inserted. We represent this sum as a sum over admissible domains $\Omega_{k+1}$, and for a fixed $\Omega_{k+1}$ we resum over admissible sets $P_{k+1}, Q_{k+1}$, i.e., the sets which satisfy all the conditions of the above constructions, and which determine the same fixed $\Omega_{k+1}$. The resummation applies only to the characteristic functions and the gauge fixing terms localized in $\Omega_{k} \backslash \Omega_{k+1}$. Denote the sum by $\zeta\left(\Omega_{k+1}^{c}\right)$. The other characteristic functions introduced above are localized in $\Omega_{k+1}$, and are equal to $\chi_{k+1}\left(\Omega_{k+1}\right) \chi_{k}^{\prime}\left(\Omega_{k+1}\right)$.

The next steps are exactly the same as in Sect. C [16], and in fact the same as in Sect. 2 [I]. We expand all the expressions with respect to $A_{k}^{\prime}$, and we linearize the expressions in the $\delta$-functions for bonds in $\Gamma_{k+1}$. Finally we remove the $\delta$-functions using the operator $C$, and we perform the scaling transformation $A_{k}^{\prime}=g_{k} A_{k}$. All the applied formulas are the same as in Sect. 2 [I], only the symbols represent different operators, determined by the sequence $\left\{\Omega_{j}\right\}$. Their properties are essentially the same as before, because of the localization of the field $A_{k}$. Let us write the result of these operations.

$$
\begin{aligned}
\left(T \varrho_{k}\right)\left(V_{k+1}\right)= & \left.\sum_{\left\{\Omega_{\jmath}\right\},\left\{A_{j}\right\}} \sum_{\Omega_{k+1}} \chi_{k+1}\left(\Omega_{k+1}\right) \int d V_{k}\right|_{\Omega_{k+1}^{c}} \delta\left(\bar{V}_{k} V_{k+1}^{-1}\right) \zeta\left(\Omega_{k+1}^{c}\right) \\
& \cdot \mathbf{T}_{k} \exp \left[A_{k}\left(\frac{1}{g_{k}^{2}}, U_{k+1}\right)+\log g_{k} d(\mathbf{g})\left|B\left(\Gamma_{k+1}\right)^{*}\right|\right. \\
& +\log \sigma_{0}\left|B\left(\Gamma_{k+1}\right)^{*}\right| \\
& \left.-\log z\left(L^{4}-1\right)\left|\Gamma_{k+1}\right|\right]\left.z^{(k)} \int d A_{k}\right|_{\Omega_{k+1}} \chi_{k}^{\prime}\left(\Omega_{k+1}\right) \\
& \cdot \exp \left[-\frac{1}{2}\left\langle A_{k}, C^{*} \Delta^{(k)} C A_{k}\right\rangle+\mathbf{P}^{(k)}\left(g_{k}, U_{k+1}, A_{k}\right)\right.
\end{aligned}
$$




$$
\begin{aligned}
& +\left\{\left(\mathbf{E}_{k}+\mathbf{R}_{k}+\mathbf{B}_{k}\right)\left(U_{k}\left(\exp i\left[g_{k} C A_{k}-h \tilde{D}\left(g_{k} C A_{k}\right)\right] V^{(k)}\right)\right)\right. \\
& -\left(\mathbf{E}_{k}+\mathbf{R}_{k}+\mathbf{B}_{k}\right)\left(U_{k+1}\right) \\
& +A\left(\frac{1}{g_{k}^{2}(\cdot)}-\frac{1}{g_{k}^{2}}, U_{k}\left(\exp i\left[g_{k} C A_{k}-h \tilde{D}\left(g_{k} C A_{k}\right)\right] V^{(k)}\right)\right) \\
& \left.\left.-A\left(\frac{1}{g_{k}^{2}(\cdot)}-\frac{1}{g_{k}^{2}}, U_{k+1}\right)\right\}\right]
\end{aligned}
$$

In the above integral the characteristic functions $\chi_{k}^{\prime}$ depend now on the field $V^{(k)}$, hence on the new field variables $V_{k+1}$ on the whole set $\Gamma_{k+1}$. We want to have characteristic functions depending on the fluctuation field only, hence we introduce another decomposition of unity. Consider the domain $\Omega_{k+1}^{\sim-1}$, i.e., the domain obtained by taking off one layer of $L M R_{k+1}$-cubes from $\Omega_{k+1}$. On this domain introduce the decomposition of unity

$$
\begin{aligned}
1 & =\sum_{R_{k+1}} \sum_{\square^{\prime}\left(R_{k+1}^{c}\right.} \chi\left(\left\{\sup _{b \in\left(\square^{\prime} \sim 2\right)^{(k) *}}\left|A_{k}(b)\right|<\delta_{k}\right\}\right) \prod_{\square^{\prime} \subset R_{k+1}} \chi\left(\left\{\sup _{b \in\left(\square^{\prime} \sim 2\right)^{(k) *}}\left|A_{k}(b)\right| \geqq \delta_{k}\right\}\right) \\
& =\sum_{R_{k+1}} \chi^{(k)}\left(R_{k+1}^{c}\right) \chi^{(k) c}\left(R_{k+1}\right),
\end{aligned}
$$

where the summation is over the subdomains of $\Omega_{k+1}^{\sim-1}$, which are unions of $L^{2} M_{2} R_{k+1}$-cubes of the partition of unity, and the complement $R_{k+1}^{c}$ means the complement of $R_{k+1}$ to the domain $\Omega_{k+1}^{\sim-1}$. For a cube $\square^{\prime} \subset R_{k+1}^{c}$ there are now two small field characteristic functions in the integral, one from the decomposition (3.3), and another from the decomposition (3.16). The first is equal to 1, because of the bound on $A_{k}$ introduced by the second, and the fact that the configuration $V^{(k)}\left(V_{\square^{\prime}}^{(k)}\right)^{-1}-1$ is small, much smaller than $\delta_{k}$. This follows from the identity analogous to $(3.6)$,

$$
\begin{aligned}
U_{k+1} & =U\left(\mathbf{B}_{k+1}\left(\square^{\prime \sim 4}\right),\left[M^{*}\left(U_{k+1}\right)\left(M^{\cdot}\left(Q_{k+1}^{s^{*}} V_{k+1}\right)\right)^{-1}\right] M^{\cdot}\left(Q_{k+1}^{s^{*}} V_{k+1}\right)\right) \\
& =\left(\exp i L^{-1} \eta \mathbf{H}_{k+1, \square^{\prime}}\left(\frac{1}{i} \log \left[M^{\cdot}\left(U_{k+1}\right)\left(M^{*}\left(Q_{k+1}^{s^{*}} V_{k+1}\right)\right)^{-1}\right]\right) U_{k+1, \square^{\prime}}\right)^{u_{k+1, \square}^{-1}} .
\end{aligned}
$$

The field $U_{k+1}$ satisfies the regularity condition $\left|\partial U_{k+1}-1\right|<2 B_{3} \varepsilon_{k+1}\left(L^{-1} \eta\right)^{2}$ on $\Omega_{k+1}$, hence the argument of the function $\mathbf{H}_{k+1, \square}$ is bounded by $44 d^{2} B_{3} \varepsilon_{k+1}$, and has a support in a boundary layer of the width $2 L M_{1}$ at the boundary of $\square^{\prime \sim 4}$. Thus, the function $\mathbf{H}_{k+1, \square^{\prime}}$ is bounded by $B_{3} \exp \left(-\delta L M_{2} R_{k+1}\right) 44 d^{2} B_{3} \varepsilon_{k+1}$ $\leqq 44 d^{2} B_{3}^{2}\left(1+\beta_{0}\right) \exp \left(-R_{k}\right) \varepsilon_{k}$ on $\square^{\prime 22}$, and

$$
V^{(k)}(b)=u_{k+1, \square^{\prime}}^{-1}\left(b_{-}\right) \exp i \widetilde{Q}_{k}\left(L^{-1} \eta \mathbf{H}_{k+1, \square^{\prime}}\right) \bar{R}^{k} u_{k+1, \square^{\prime}}\left(b_{+}\right) V_{\square^{\prime}}^{(k)}(b) \text {. }
$$

These facts, and the bounds (106)-(108), (159)-(163) in [12], imply

$$
\begin{aligned}
\left|V^{(k)}(b)\left(V_{\square^{\prime}}^{(k)}(b)\right)^{-1}-1\right| & <O(1) 44 d^{2} B_{3}^{2}\left(1+\beta_{0}\right) \varepsilon_{k} \exp \left(-R_{k}\right) \\
& =O(1) 44 d^{2} B_{3}^{2}\left(1+\beta_{0}\right) \frac{A_{0}}{A_{1}} \exp \left(-R_{k}\right) \delta_{k}<\delta_{k}
\end{aligned}
$$


for $g_{k}$, or $\gamma$ sufficiently small, and $b \in\left(\square^{\prime \sim 2}\right)^{(k)^{*}}$. Thus for cubes $\square^{\prime} \subset R_{k+1}^{c}$ there are only the small field characteristic functions from the decomposition (3.16) in the integral. The domain $R_{k+1}^{\prime}$ is defined, as usual, as a union of the $L M R_{k+1}$-cubes intersecting $R_{k+1}$. We surround the domain $\left(\Omega_{k+1}^{c}\right)^{\sim} \cup R_{k+1}^{\prime}$ by a layer of $L M R_{k+1}$ cubes, and we denote the complement of the so-obtained domain by $\Lambda_{k+1}$, hence

$$
\begin{aligned}
& \Lambda_{k+1}=\left(\left(\Omega_{k+1}^{c}\right)^{\sim 2} \cup R_{k+1}^{\prime}\right)^{c}=\Omega_{k+1}^{\sim-2} \cap\left(R_{k+1}^{\prime \sim}\right)^{c}, \\
& S_{k+1}=\left(\Omega_{k+1} \backslash \Omega_{k+1}^{\sim-1}\right) \cup R_{k+1} .
\end{aligned}
$$

In fact we modify the domain $\Lambda_{k+1}$ in the same way as in Sect. 1 ; we consider components of $\Lambda_{k+1}^{c}$, and if a component is contained in a cube of the size $100 L M R_{k+1}$, then we replace it by the smallest rectangular parallelepiped containing it. On the domain $\Lambda_{k+1}$, in fact on $\Lambda_{k+1}^{\sim}$ also, there are only the small field characteristic functions from (3.16) in the integral, their product is $\chi^{(k)}\left(\Lambda_{k+1}\right)$. On the domain $\Omega_{k+1} \cap \Lambda_{k+1}^{c}$ there is the function

$$
\chi\left(\Omega_{k+1} \cap \Lambda_{k+1}^{c}, S_{k+1}\right)=\chi^{(k)}\left(R_{k+1}^{c} \cap \Lambda_{k+1}^{c}\right) \chi^{(k) c}\left(R_{k+1}\right) \chi_{k}^{\prime}\left(S_{k+1}\right) .
$$

The last function above depends nonlocally on $V_{k+1}$, through the function $V^{(k)}$. We remove this dependence by an operation similar to the one in Sect. 1, formulas (1.21)-(1.23). We take the determining set $\mathbf{B}\left(\Lambda_{k+1}^{c} \cap \Lambda_{k}\right) \cup \mathbf{B}_{k+1}$ defined in (2.14), which in this case is simply equal to the union $\left.\left.\mathbf{B}_{k+1}\left(\Lambda_{k+1}^{c}\right)\right|_{\Omega_{k+1}} \cup \mathbf{B}_{k}\left(\Lambda_{k}\right)\right|_{\Omega_{k+1}^{c}}$, and the corresponding function

$$
\begin{aligned}
& U_{k+1, \Lambda_{k+1}^{c} \cap \Lambda_{k}}(V)=U\left(\mathbf{B}\left(\Lambda_{k+1}^{c} \cap \Lambda_{k}\right) \cup \mathbf{B}_{k+1},\right. \\
& \left.\quad\left(\left.M^{\cdot}\left(Q_{k+1}^{s^{*}} V_{k+1}\right)\right|_{\Lambda_{k+1}^{c} \cap \Omega_{k+1}},\left.M^{\cdot}\left(Q_{k}^{s^{*}} V_{k}\right)\right|_{\Lambda_{k} \cap \Omega_{k+1}}\right)\right) .
\end{aligned}
$$

We want to replace $V^{(k)}$ by the $k$-th average of the above function. We use again the formula (3.17), but with $U_{k+1, \square}$, replaced by the above function. It yields the representation (3.18) with the corresponding changes, e.g., $V_{\square}^{(k)}$ is replaced by $V_{\Lambda_{k+1}^{c}\left(\Lambda_{\Lambda_{k}}\right.}^{(k)}$, and with similar bounds. Denoting

$$
V^{(k)}\left(V_{\Lambda_{k+1}^{c} \cap \Lambda_{k}}^{(k)}\right)^{-1}=\exp i \mathbf{H}^{(k)},
$$

we obtain that $\mathbf{H}^{(k)}$ is an analytic function of the background field $U_{k+1}$ restricted to $\Lambda_{k+1}^{c} \cap \Lambda_{k}$, bounded on the set $S_{k+1}$ by $O(1) \varepsilon_{k} \exp \left(-R_{k}\right)$, hence by any positive power of $g_{k}$. Now we make a change of variables for each bond variable $A_{k}(b), b \in S_{k+1}^{(k)^{*}}$. For bonds in $\left(R_{k+1}^{\sim 2} \cap S_{k+1}\right)^{(k)^{*}}$ we take the change of variables given by the formula (1.22), with $\mathbf{H}^{(k)}(b)$ instead of $\mathbf{H}_{1, \mathbf{A x}}(b)$. For the remaining bonds we take the simpler change of variables with the function $g\left(A_{k}^{\prime}(b)\right)$ replaced by 1 . These changes of variables transform the function $\chi_{k}^{\prime}\left(S_{k+1}\right)$ into the same function, but with $V^{(k)}$ replaced by $V_{\Lambda_{k+1}^{c}}^{(k)} \cap \Lambda_{k}$. We use the same notation for the new function. The other characteristic functions are unchanged. The action is changed by terms corresponding to (1.24), and by terms obtained by the first order expansion of the action with respect to the perturbative parts of the changes of variables. We denote this new part of the action by $V^{(k)}\left(S_{k+1}, A_{k}, \mathbf{H}^{(k)}\right)$. All terms of the previous action contribute to it, but the differentiation with respect to the perturbation gives an additional localization in $S_{k+1}$. The above operations render each term of the sum 
in (3.15) as a sum of terms. We write this sum as a sum over domains $\Lambda_{k+1}$, and for a fixed domain $\Lambda_{k+1}$, as a sum over the admissible sets $S_{k+1}$.

For each term of the obtained sum we consider the conditional integral with respect to $A_{k}$ restricted to $A_{k+1}$. The remaining integration with respect to $A_{k}$ restricted to $\Omega_{k+1} \cap \Lambda_{k+1}^{c}$ is included into the operation $\mathbf{T}^{(k+1)}$. Thus we define

$$
\begin{aligned}
\mathbf{T}^{(k+1)}= & \left.\left.\sum_{S_{k+1}} \int d V_{k}\right|_{\Omega_{k+1}^{c}} \delta\left(\bar{V}_{k} V_{k+1}^{-1}\right) \zeta\left(\Omega_{k+1}^{c}\right) z^{(k)} \int d A_{k}\right|_{\Omega_{k+1} \cap \Lambda_{k+1}^{c}} \chi\left(\Omega_{k+1} \cap \Lambda_{k+1}^{c}, S_{k+1}\right) \\
& \cdot \exp \left[-\frac{1}{2}\left\langle A_{k}, C^{*} \Delta^{(k)} C A_{k}\right\rangle+\frac{1}{2}\left\langle A_{k}, C^{*} \Delta^{(k)} C C^{(k)}\left(\Lambda_{k+1}\right) C^{*} \Delta^{(k)} C A_{k}\right\rangle\right] .
\end{aligned}
$$

Here $z^{(k)}$ denotes the product of factors corresponding to bonds in $\Gamma_{k+1} \cap \Lambda_{k+1}^{c}$. Denoting the sum of constants in the first exponential in (3.15) by $E_{0}^{(k)}$, and defining

$$
\mathbf{T}_{k+1}=\mathbf{T}^{(k+1)} \mathbf{T}_{k},
$$

we get

$$
\begin{aligned}
\left(T \varrho_{k}\right)\left(V_{k+1}\right)= & \sum_{\left\{\Omega_{j}\right\},\left\{V_{j}\right\}} \chi_{k+1}\left(\Omega_{k+1}\right) \mathbf{T}_{k+1} \exp \left[A_{k}\left(\frac{1}{g_{k}^{2}}, U_{k+1}\right)+E_{0}^{(k)}\right] \\
& \left.\cdot z^{(k)} \int d A\right|_{\Lambda_{k+1}} \chi^{(k)}\left(\Lambda_{k+1}\right) \exp \left[-\frac{1}{2}\left\langle A, C^{*} \Delta^{(k)} C A\right\rangle\right. \\
& -\left\langle A, C^{*} \Delta^{(k)} C A_{k}\right\rangle \\
& -\frac{1}{2}\left\langle A_{k}, C^{*} \Delta^{(k)} C C^{(k)}\left(\Lambda_{k+1}\right) C^{*} \Delta^{(k)} C A_{k}\right\rangle \\
& \left.+\mathbf{P}^{(k)}\left(g_{k},\left(A_{k}, A\right)\right)+\{\ldots\}+V^{(k)}\left(S_{k+1},\left(A_{k}, A\right)\right)\right] .
\end{aligned}
$$

Here $A_{k}$ denotes the fluctuation field on $\Omega_{k+1} \cap \Lambda_{k+1}^{c}$, and $A$ denotes this field on $\Lambda_{k+1}$.

Now the fundamental problem is to represent the logarithm of the last fluctuation field integral in the form described by the inductive assumption, i.e. as the sum of three terms $\mathbf{E}^{(k+1)}+\mathbf{R}^{(k+1)}+\mathbf{B}^{(k+1)}$ satisfying the inductive assumption. Let us remark that these terms correspond to the particular sequences $\left\{\Omega_{j}\right\},\left\{\Lambda_{j}\right\},\left\{S_{j}\right\}$ obtained after the $k+1$-st renormalization transformation, and they will be changed properly after the $\mathbf{R}$-operation. Therefore we need the improved statements and bounds for these terms, discussed before the formulation of Theorem 1, but we obtain such improvements quite naturally.

At first we decompose the logarithm of the fluctuation field integral into a sum of three expressions, which contribute mainly to the three terms. This decomposition is rather naturally connected with the form of the fluctuation field effective action in (3.15), but we divide the expression determined by $\mathbf{R}_{k}$ into two parts, and we treat them differently. Let us recall that the Euclidean covariance property (2.32) holds for Euclidean transformations leaving invariant the partition of the lattice $T_{\eta}$ into $L^{-(k-j)} M R_{j}$-cubes. For $k-j$ large enough, more precisely for $j \leqq k_{1}$, where $k_{1}$ is the largest integer satisfying $L^{-\left(k-k_{1}\right)} M R_{j} \leqq L$, the cubes of the partition are 
contained in $L$-cubes corresponding to the $L$-blocks. Therefore the expression determined by the sum $\mathbf{R}_{k}^{\prime}=\sum_{j=1}^{k_{1}} \mathbf{R}^{(j)}$ is Euclidean covariant with respect to the Euclidean transformations leaving invariant the lattice $T^{(k+1)}$. It has the same covariance property as the expression determined by $\mathbf{E}_{k}$, and we include it into $\mathbf{E}_{k}$. The sum of the two expressions, $\mathbf{R}_{k}^{\prime}$ and $\mathbf{E}_{k}$, is denoted also by $\mathbf{E}_{k}$. The sum of the remaining terms $\mathbf{R}_{k}^{\prime \prime}=\sum_{j=k_{1}+1}^{k} \mathbf{R}^{(j)}$ is treated separately. Introduce now auxiliary parameters $s, t$, the parameter $t$ multiplying the expressions $\mathbf{B}_{k}, A\left(\left(1 / g_{k}^{2}(\cdot)\right)\right.$ $\left.-\left(1 / g_{k}^{2}\right), \ldots\right), V^{(k)}, s$ multiplying $\mathbf{R}_{k}^{\prime \prime}$. We have

$$
\begin{aligned}
\log \left[z^{(k)} \int d A \chi^{(k)} \exp \left[-\frac{1}{2}\left\langle A, C^{*} \Delta^{(k)} C A\right\rangle-\ldots\right]\right] \\
=\int_{0}^{1} d t\left\langle\left\{\mathbf{B}_{k}\left(U_{k}(\ldots)\right)-\mathbf{B}_{k}\left(U_{k+1}\right)+A\left(\frac{1}{g_{k}^{2}(\cdot)}-\frac{1}{g_{k}^{2}}, U_{k}(\ldots)\right)\right.\right. \\
\left.\left.\quad-A\left(\frac{1}{g_{k}^{2}(\cdot)}-\frac{1}{g_{k}^{2}}, U_{k+1}\right)\right\}+V^{(k)}\left(S_{k+1}\right)\right\rangle_{s=1, t} \\
\quad+\int_{0}^{1} d s\left\langle\left\{\mathbf{R}_{k}^{\prime \prime}\left(U_{k}(\ldots)\right)-\mathbf{R}_{k}^{\prime \prime}\left(U_{k+1}\right)\right\}\right\rangle_{s, t=0} \\
\quad+\log \left[z^{(k)} \int d A \chi^{(k)} \exp \left[-\frac{1}{2}\left\langle A, C^{*} \Delta^{(k)} C A\right\rangle-\ldots(s=0, t=0) \ldots\right]\right] .
\end{aligned}
$$

Here $\langle\cdot\rangle_{s, t}$ denotes the expectation value with respect to the probabilistic measure

$$
Z_{s, t}^{-1} d \mu_{C^{(k)}\left(\Lambda_{k+1}\right)}(A) \chi_{k} \exp \left[-\left\langle A, C^{*} \Delta^{(k)} C A_{k}\right\rangle+\mathbf{P}^{(k)}+\{\ldots\}_{s, t}+t V^{(k)}\left(S_{k}\right)\right],
$$

where the introduction of the parameters $s, t$ was described above, and $Z_{s, t}$ is the normalization factor.

On the right-hand side of (3.6) we have the sum of three expressions. The first is the integral over $t$ of the expectation value of the boundary terms, and it contributes to $\mathbf{B}^{(k+1)}$ only. The second is the integral over $s$ of the expectation value of the $R$-terms, and it contributes to $\mathbf{R}^{(k+1)}$ and $\mathbf{B}^{(k+1)}$. The third expression is the logarithm of the fluctuation field integral involving the regular terms of the effective action only, and it contributes to $\mathbf{E}^{(k+1)}$ and $\mathbf{B}^{(k+1)}$. This expression has to be transformed further, because we need the particular representation (2.26), (2.27) for the regular terms. At first we separate obvious boundary terms connected with the external fluctuation field $A_{k}$, by expanding in this field. We introduce the parameter $t$ multiplying $A_{k}$ in the expressions in the logarithm. We have

$$
\begin{gathered}
\log \left[z^{(k)} \int d A \chi^{(k)} \exp \left[-\frac{1}{2}\left\langle A, C^{*} \Delta^{(k)} C A\right\rangle-\ldots(s=0, t=0) \ldots\right]\right] \\
\quad=\int_{0}^{1} d t\left\langle-\left\langle A, C^{*} \Delta^{(k)} C A_{k}\right\rangle+\left\langle\frac{\delta}{\delta t A_{k}} \mathbf{P}^{(k)}\left(g_{k},\left(t A_{k}, A\right)\right), A_{k}\right\rangle\right.
\end{gathered}
$$




$$
\begin{aligned}
& \left.+\left\langle\frac{\delta}{\delta t A_{k}} \mathbf{E}_{k}\left(U_{k}(\ldots)\right), A_{k}\right\rangle\right\rangle_{t}-\frac{1}{2}\left\langle A_{k}, C^{*} \Delta^{(k)} C C^{(k)}\left(\Lambda_{k+1}\right) C^{*} \Delta^{(k)} C A_{k}\right\rangle \\
& +\log \left[z^{(k)} \int d A \chi^{(k)} \exp \left[-\frac{1}{2}\left\langle A, C^{*} \Delta^{(k)} C A\right\rangle-\ldots\left(s=0, t=0, A_{k}=0\right) \ldots\right]\right] \\
& =\int_{0}^{1} d t\left\langle-\left\langle A_{k}, C^{*} \Delta^{(k)} C C^{(k)}\left(\Lambda_{k+1}\right)\left(\frac{\delta}{\delta A} \chi^{(k)}+\frac{\delta}{\delta A} \mathbf{P}^{(k)}\left(g_{k},\left(t A_{k}, A\right)\right)\right.\right.\right. \\
& \left.\left.+\frac{\delta}{\delta A} \mathbf{E}_{k}\left(U_{k}(\ldots)\right)\right)\right\rangle \\
& \left.+\left\langle\frac{\delta}{\delta t A_{k}} \mathbf{P}^{(k)}\left(g_{k},\left(t A_{k}, A\right)\right), A_{k}\right\rangle+\left\langle\frac{\delta}{\delta t A_{k}} \mathbf{E}_{k}\left(U_{k}(\ldots)\right), A_{k}\right\rangle\right\rangle_{t} \\
& +\log \left[z ^ { ( k ) } \int d A \chi ^ { ( k ) } \operatorname { e x p } \left[-\frac{1}{2}\left\langle A, C^{*} \Delta^{(k)} C A\right\rangle+\mathbf{P}^{(k)}\left(g_{k}, A\right)\right.\right. \\
& \left.+\left\{\mathbf{E}_{k}\left(U_{k}\left(\exp i\left[g_{k} C A-h \tilde{D}\left(g_{k} C A\right)\right] V^{(k)}\right)\right)-\mathbf{E}_{k}\left(U_{k+1}\right)\right\}\right]
\end{aligned}
$$

Here $\langle\cdot\rangle_{t}$ denotes the expectation value with respect to the probabilistic measure

$$
\begin{aligned}
Z_{t}^{-1} d \mu_{C^{(k)}\left(A_{k+1}\right)}(A) \chi^{(k)} \exp \left[-t\left\langle A, C^{*} \Delta^{(k)} C A_{k}\right\rangle\right. \\
\left.\quad+\mathbf{P}^{(k)}\left(g_{k},\left(t A_{k}, A\right)\right)+\left\{\mathbf{E}_{k}\left(U_{k}\left(\ldots\left(t A_{k}, A\right) \ldots\right)\right)-\mathbf{E}_{k}\left(U_{k+1}\right)\right\}\right] .
\end{aligned}
$$

The first expression on the right-hand side of (3.28) is the expectation value of the sum of terms with at least one localization in the domain $\Omega_{k+1} \backslash \Lambda_{k+1}$, hence this expression contributes to $\mathbf{B}^{(k+1)}$ only.

The remaining expression in (3.28), the last logarithm, is formally given by the same formula as the term $\mathbf{E}^{(k+1)}$ in [I] [see (2.12), (2.13)], but the integral is restricted to $\Lambda_{k+1}$, and operators are determined by the sequence $\left\{\Omega_{j}\right\}$. To get the representation (2.26), (2.27) we expand this expression with respect to $g_{k}$ up to the first order. The idea is that this expansion is obtained by expanding the underintegral expression in $g_{k} C A$, because of the special structure of these expressions. The field $g_{k} C A$ has the desired dependence on space variables (the bond variables in this case), which can be used to construct (2.26). Multiplying $g_{k}$ by the parameter $t$ we have

[the logarithm on the right-hand side of (3.28)]

$$
\begin{aligned}
= & \log \left[z^{(k)} \int d A \exp \left[-\frac{1}{2}\left\langle A, C^{*} \Delta^{(k)} C A\right\rangle\right]\right]+\log \int d \mu_{C^{(k)}\left(\Lambda_{k+1}\right)} \chi^{(k)} \\
& +g_{k} \int_{0}^{1} d t\left\langle\frac{\partial}{\partial t g_{k}} \mathbf{P}^{(k)}\left(\operatorname{tg}_{k}, A\right)+\frac{\partial}{\partial \operatorname{tg}_{k}} \mathbf{E}_{k}\left(U_{k}\left(\ldots \operatorname{tg}_{k} C A \ldots\right)\right)\right\rangle_{t},
\end{aligned}
$$

where the expectation value is with respect to the probabilistic measure defined by the function $\chi^{(k)} \exp \left[\ldots t g_{k} \ldots\right]$ in the logarithm, but with the constant $g_{k}$ replaced by $t g_{k}$. Let us now analyze successively the terms in the above expansion. Consider the 
derivatives in the last term, in the expectation value. They can be expressed as inner products of functional derivatives of some functions of $\operatorname{tg}_{k} C A$, with the field $C A$. This is obvious for all terms in the effective action, as displayed in the formula (2.12) [I], maybe with the exception of the terms divided by $\left(\operatorname{tg}_{k}\right)^{2}$. These we expand up to the second order with respect to $\operatorname{tg}_{k} C A$. We use the fact that the lower-order terms vanish, and we represent the remainders by quadratic forms in $A$, as in (6.42) [I]. The coefficients are functions of $\operatorname{tg}_{k} C A$, and the differentiation with respect to $\operatorname{tg}_{k}$ yields such an inner product. Thus the derivatives in the expectation value can be written as

$$
\left\langle\mathbf{V}_{k}^{\prime}\left(\operatorname{tg}_{k}, A\right), C A\right\rangle=\sum_{b \in \Lambda_{k+1}^{(k)}} \operatorname{tr} \mathbf{V}_{k}^{\prime}\left(\operatorname{tg}_{k}, A, b\right)(C A)(b),
$$

where $\mathbf{V}_{k}^{\prime}$ is the sum of all the functional derivatives. Consider the second logarithm on the right-hand side of (3.30). Denote the characteristic function with the parameter $t$ multiplying the variables $A$ by $\chi_{t}^{(k)}$. Thus there is the function $\chi_{1}^{(k)}$ in this expression, and $\chi_{0}^{(k)}=1$. We have

$$
\log \int d \mu_{C^{(k)}\left(\Lambda_{k+1}\right)} \chi^{(k)}=\int_{0}^{1} d t \int d \mu_{C^{(k)}\left(\Lambda_{k+1}\right)} \frac{\partial}{\partial t} \chi_{t}^{(k)}\left(\int d \mu_{C^{(k)}\left(\Lambda_{k+1}\right)} \chi_{t}^{(k)}\right)^{-1},
$$

and $(\partial / \partial t) \chi_{t}^{(k)}$ can be written as a sum of terms, for which only one characteristic function in the product is differentiated. Consider now the first logarithm on the right-hand side of (3.30). The factor $z^{(k)}$ comes from elimination of the $\delta$-functions, and is equal to the product of the factors $z^{(k)}(c)$ for $c \in \Lambda_{k+1}^{(k+1)}$. We have

$$
\begin{aligned}
& \log \left[z^{(k)} \int d A \exp \left[-\frac{1}{2}\left\langle A, C^{*} \Delta^{(k)} C A\right\rangle\right]\right] \\
& \quad=\sum_{c \in A_{k+1}^{(k+1)}} \log z^{(k)}(c)-\frac{1}{2} \log \operatorname{det}\left(C^{*} \Delta^{(k)} C\right)+\log \int d A \exp \left[-\frac{1}{2}|A|^{2}\right] .
\end{aligned}
$$

The last term is a number, which contributes to the vacuum energy renormalization, so we include it into the definition of $E_{0}^{(k)}$. The first term has already the localized form, so we consider the second term. We have

$$
\begin{aligned}
-\frac{1}{2} \log \operatorname{det}\left(C^{*} \Delta^{(k)} C\right) & =-\frac{1}{2} \operatorname{Tr} \log \left(C^{*} \Delta^{(k)} C\right) \\
& =\frac{1}{4 \pi i} \int_{\gamma} d z \log z \operatorname{Tr}\left(C^{*} \Delta^{(k)} C-z I\right)^{-1},
\end{aligned}
$$

where the contour $\gamma$ surrounds the spectrum of $C^{*} \Delta^{(k)} C$, e.g. we take $\gamma$ composed of a segment of the circle $|z|=R$ for $R$ large enough, and of an interval on the line $\operatorname{Re} z=r, r$ positive and small. We take $\lambda_{0}>r$, but small enough, and we expand the resolvent in (3.34) as follows:

$$
\left(C^{*} \Delta^{(k)} C-z I\right)^{-1}=\left(\lambda_{0}-z\right)^{-1} I-\left(\lambda_{0}-z\right)^{-1}\left(C^{*} \Delta^{(k)} C-\lambda_{0} I\right)\left(C^{*} \Delta^{(k)} C-z I\right)^{-1} .
$$


The integral of the first term on the right-hand side is equal to $-1 / 2 \log \lambda_{0} I$. The second term is $O\left(|z|^{-2}\right)$ as $|z| \rightarrow \infty$, hence we can replace the contour of integration by the contour $\{z: \operatorname{Im} z=-r, \operatorname{Re} z \leqq 0\} \cup\{z: \operatorname{Re} z=0,|\operatorname{Im} z| \leqq r\} \cup\{z: \operatorname{Im} z=r$, $\operatorname{Re} z \leqq 0\}$, and next take the limit as $r \rightarrow 0$. This yields

$$
\begin{aligned}
-\frac{1}{2} \log \operatorname{det}\left(C^{*} \Delta^{(k)} C\right)= & -\frac{1}{2} \log \lambda_{0}\left|\Lambda_{k+1}^{(k) *}\right| \\
& +\frac{1}{2} \int_{0}^{\infty} d \lambda\left(\lambda_{0}+\lambda\right)^{-1} \operatorname{Tr}\left(C^{*} \Delta^{(k)} C-\lambda_{0} I\right)\left(C^{*} \Delta^{(k)} C+\lambda I\right)^{-1} .
\end{aligned}
$$

Let us introduce the following definiton:

$$
\begin{aligned}
\mathbf{E}_{0}^{(k+1)}\left(\Lambda_{k+1}, U_{k+1}, b\right)= & \chi_{\Lambda_{k^{(k)}}(k)}(b)\left[-\frac{1}{2} \log \lambda_{0}+\frac{1}{2} \int_{0}^{\infty} d \lambda\left(\lambda_{0}+\lambda\right)^{-1}\right. \\
& \cdot \operatorname{tr}\left(\left(C^{*} \Delta^{(k)} C-\lambda_{0} I\right)\left(C^{*} \Delta^{(k)} C+\lambda\right)^{-1}\right)(b, b) \\
& +\int_{0}^{1} d t \int d \mu_{C^{(k)}\left(\Lambda_{k+1}\right)} \prod_{b^{\prime} \in \Lambda_{k+1}^{(k) *}, b^{\prime} \neq b} \chi^{(k)}\left(t A\left(b^{\prime}\right)\right) \frac{\partial}{\partial t} \chi^{(k)}(t A(b)) \\
& \left.\cdot\left(\int d \mu_{C^{(k)}\left(\Lambda_{+1}\right)} \chi_{t}^{(k)}\right)^{-1}\right] \\
& +g_{k} \int_{0}^{1} d t\left\langle\operatorname{tr} \mathbf{V}_{k}^{\prime}\left(t g_{k}, A, b\right)(C A)(b)\right\rangle_{t} .
\end{aligned}
$$

The identities (3.30)-(3.33), (3.36), together with the above definition, imply the equality

[the logarithm on the right-hand side of (3.28)]

$$
=\sum_{c \in \Lambda_{k+1}^{(k+1)}} \log z^{(k)}(c)+\sum_{b \in \Lambda_{k+1}^{(k)}} \mathbf{E}_{0}^{(k+1)}\left(\Lambda_{k+1}, b\right)+\text { const }
$$

The constant above is the constant in (3.33), included into the vacuum renormalization $E_{0}^{(k)}$.

The terms in (3.38) are gauge invariant. If they are constructed assuming that $\Lambda_{j+1}$ is the whole space, i. e. assuming $\Omega_{j}=\Lambda_{j}=T_{\eta}$ for $j=1, \ldots, k+1$, then they are also Euclidean covariant, and we have

$$
\mathbf{E}^{(k+1)}\left(r U_{k+1}, r^{-1} b\right)=\mathbf{E}^{(k+1)}\left(U_{k+1}, b\right) .
$$

Here $r$ is an arbitrary Euclidean transformation of the continuous space $T$, transforming the lattice $T^{(k+1)}$ into itself. We have dropped the subscript 0 because in this case the expressions (3.37) coincide with the expressions in the inductive assumption, e.g. in (2.26), (2.27). The Euclidean covariance (3.39) follows from the transformation laws (2.29), (2.32), and from the definitions of the expressions in (3.37). 
Next we introduce functions $h_{z}$ on the lattice $T_{1}^{(k)}$, for $z \in T_{L}^{(k+1)}$ :

$$
h_{z}(x)=\prod_{\mu=1}^{d} h\left(x_{\mu}-z_{\mu}\right), \quad h(t)=\max \left\{1-L^{-1}|t|, 0\right\} .
$$
They form a decomposition of unity: $\sum_{z} h_{z}=1$. With the help of these functions
we define

$$
\begin{aligned}
\mathbf{E}_{0}^{(k+1)}\left(\Lambda_{k+1}, U_{k+1}, z\right)= & \sum_{\mu=1}^{d}\left[\frac{1}{2}\left(\log z^{(k)}\left(\left\langle z-L e_{\mu}, z\right\rangle\right)+\log z^{(k)}\left(\left\langle z, z+L e_{\mu}\right\rangle\right)\right)\right. \\
+ & \left.\sum_{x} h_{z}(x) \mathbf{E}_{0}^{(k+1)}\left(\Lambda_{k+1},\left\langle x, x+e_{\mu}\right\rangle\right)\right] \\
& z \in \Lambda_{k+1}^{(k+1)} .
\end{aligned}
$$

This function has the same properties as the function (3.37), namely it is gauge invariant, and, when defined on the whole lattice, it is Euclidean covariant, i.e. it satisfies the inductive assumption (2.29) for $j=k+1$. The equality (3.38) implies

[the logarithm on the right-hand side of (3.28)] $=\sum_{z \in \Lambda_{k+1}^{(k+1)}} \mathbf{E}_{0}^{(k+1)}\left(\Lambda_{k+1}, z\right)+$ const .

The functions $\mathbf{E}_{0}^{(k+1)}(z)$ are not equal yet to the functions $\mathbf{E}^{(k+1)}(z)$ in the inductive assumptions. These are obtained by constructing localized expansions of the expressions in (3.41), and summing up terms with localizations in $\Lambda_{k+1}$. The localized expansions are cluster expansions for the expectation values, and the generalized random walk expansions for the operators in the second term on the right-hand side of (3.37).

Let us consider now the expectation values in the formulas (3.26), (3.28), (3.37). To construct their cluster expansions we repeat all the considerations of Sect. 3.7 [I], with minor changes connected with the boundary layers $\left\{\Omega_{j} \backslash \Omega_{j+1}\right\}$, and additions connected with the new terms in the effective action. We describe here briefly these changes and additions only. Let us start with the last expectation value in (3.37), and more exactly with the analysis of the effective action given by the expression in the last exponential in (3.28). Formally it is equal to the effective action in (2.12), (2.13) [I], but the fluctuation field $A$ is localized in $\Lambda_{k+1}^{(k) *}$, and, what is more important, all the operators and the background field configuration $U_{k+1}$ are determined by the sequence of domains $\left\{\Omega_{j}\right\}$. The difference in the analysis is connected with the fact that on the domain $\Omega_{j} \backslash \Omega_{j+1}$ the configuration $U_{k+1}$ coincides with a $U_{j}$-type configuration, it has the same regularity properties and bounds. Therefore we have to change properly the scales on this domain. In [I] we have constructed the family of cubes $\{\square\}$ covering the space $T$, and the corresponding partition of unity $\left\{\zeta_{\square}\right\}$. Now we take the family of these cubes covering the domain $\Omega_{k}$, and for each layer $\Omega_{j} \backslash \Omega_{j+1}$ we construct its cover by cubes $\square$, which are unions of $2^{d}$ neighboring cubes from $\pi_{j}$, i.e. cubes of the size $2 M L^{j} \eta$ (measured in $\eta$-scale). For the union of these families, which is a cover of $\Omega_{1}$, we construct the corresponding partition of unity. Such covers and partitions were constructed and used in $[11,13]$, in connection with the generalized random walk expansions. 
We repeat the analysis of Sect. $3[\mathrm{I}]$ using the above cover $\{\square\}$, and the partition of unity $\left\{\zeta_{\square}\right\}$. For the term $\mathbf{E}^{(j)}$ it is in fact restricted to the domain $\Lambda_{j}$. If a cube $\square$ in the expansion (I.3.4) is a union of $2^{d}$ cubes from $\pi_{n}, j \leqq n \leqq k$, then the operation $\sim$ is also defined using cubes from $\pi_{n}$. Thus the conditions (I.3.5) have a different meaning now, the difference shows up in bounds only. In all bounds connected with such a cube we have to replace $\eta=L^{-k}$ by $L^{-n}$. For bounds involving distances it is obvious, e.g. if a localization domain $X \in \mathbf{D}_{j}$ is disjoint with $\square^{\sim}$, then $\operatorname{dist}(X, \square)>M L^{n} \eta$, hence in the $\xi$-scale $\operatorname{dist}^{(\xi)}(X, \square)>M\left(L^{j} L^{-n}\right)^{-1}$. Other bounds involve regularity properties of the configuration $U_{k+1}$ on a neighborhood of $\Omega_{n} \backslash \Omega_{n+1}$, and these are the same as for $U_{n}$-configurations, i.e. they are characterized by the scale $\mathrm{L}^{-n}$. Thus, for terms connected with such a cube $\square$, we obtain all the formulas and bounds of Sects. 3, 4 [I] with $\eta$ replaced by $\mathrm{L}^{-n}$. In fact we have better bounds, because the fluctuation field is localized in $\Omega_{k+1}$, and the exponential decay of propagators and minimizing functions yields additional small factors. They contribute to the bound (2.38), because such terms will be included into boundary terms.

After the completion of the operations of Sects. 3-5[I] we localize the obtained expressions. We repeat again the analysis of Sect. 6 [I], with the only change that the random walk expansions we use now are constructed for the sequence $\left\{\Omega_{j}\right\}$, so we apply Theorems 3.7-3.10 [13] in their full generality. The expansions (6.9) [I] are constructed for families $\sigma_{0}=\{\Delta\}$ of cubes in proper scales, i.e. if $\Delta \subset \Omega_{j} \backslash \Omega_{j+1}$, then the size of $\Delta$ is equal to $M L^{j} \eta$. Then we resum them in localization domains $Y_{0} \in \mathbf{D}_{k}$, and we obtain the expansions (I.6.10) with these domains. The remaining considerations and bounds are the same as in [I]. The same remarks apply to the expression $\mathbf{P}^{(k)}$, and we obtain Lemmas 5, 6 [I] with the corresponding changes. More precisely, the terms of the expansion (I.6.41), with localization domains $Y$ contained in $\Lambda_{k+1}$, satisfy exactly all the conditions of those lemmas. The remaining terms satisfy such conditions also, but with analyticity space (I.6.34) replaced by the space

$$
\tilde{U}_{k+1}^{c}\left(Y,(1+\beta) \tilde{\alpha}_{0},(1+\beta) \tilde{\alpha}_{1}\right) \times\left\{A: \operatorname{supp} A \subset Y \cap \Lambda_{k+1}^{(k) *},|A|<C_{1} p_{1}\left(g_{k}\right)\right\} .
$$

Notice also that the localization domains occurring in this expansion have nonempty intersections with $\Lambda_{k+1}$. These remaining terms will contribute to the boundary terms $\mathbf{B}^{(k+1)}$ in the final expansion.

Let us discuss briefly the above operations for the actions defining the measures (3.27), (3.29). The action in (3.29) differs only by the additional dependence on the field $t A_{k}$, but this was already taken into acount in the above considerations. The action in (3.27) contains more expressions. The expression determined by $\mathbf{R}_{k}$ is treated in exactly the same way as $\mathbf{E}_{k}$, except the second renormalization connected with the coupling constant renormalization counterterm, which is not needed here. In fact the analylsis presented in [16] for three-dimensional models is enough here. The expression determined by the boundary terms $\mathbf{B}_{k}$ is much simpler to deal with. To its terms we apply the formulas (3.6), (3.7) [I], and next we localize the obtained expressions. The localized expansion is over domains $Y_{0} \in \mathbf{D}_{k}$ having nonempty intersections with $\Lambda_{k+1}$. Terms of this expansion can be extended [as in (I.3.13)] to analytic functions defined on the corresponding spaces (3.43). Notice that on the 
domain $\Omega_{k+1}^{c}$ there is no regularity improvement of the configuration $U_{k+1}$; on the other hand we have to use a part of the analyticity domains of terms in $\mathbf{B}_{k}$ for the function $\mathbf{H}_{k}$. This is the reason for putting the powers of $1 / 2$ in the conditions (2.36)-(2.39). The analyticity domains become smaller after each step, but the difference is very small and exponentially decreasing in the number of steps. The localization of the fluctuation field, and the exponential decay of the minimizing function $\mathbf{H}_{k}$ imply that this difference is still much greater than bounds on this function. The terms of the expansion can be estimated as in (2.42), but with the additional factor $O\left(\varepsilon_{k}\right)$ arising from the bound of the fluctuation field. The last expression in the curly bracket in (3.27), or in (3.15), is at first localized by the decomposition of unity $\left\{\zeta_{\square}\right\}$ introduced into the sum over plaquettes in the Wilson action. We obtain a sum of terms localized in $Z_{k}$, because $\operatorname{supp}\left(g_{k}^{-2}(\cdot)-g_{k}^{-2}\right)$ $\subset Z_{k}$. These terms contribute to the boundary terms only, and they are treated as above. Notice that the large values of the function $g_{k}^{-2}(\cdot)-g_{k}^{-2}$ are suppressed by the exponential decay of $\mathbf{H}_{k}$. Finally, the expression $V^{(k)}\left(S_{k+1}\right)$ arises as the sum of all the expressions discussed above, but expanded with respect to the perturbative terms in the transformations (1.22). These expressions have at least one localization in $S_{k+1}$, and are treated again as the boundary terms.

The expressions in the expectation values in (3.26), (3.28), (3.37) are expanded and localized in the same way as the corresponding expressions in the actions discussed above.

The final step in constructing localized expansions of the expectation values is the exponentiated cluster expansion of Sect.7[I], or rather its appropriate generalization. Let us start the construction for the last expectation value in (3.37) again. The normalization factor in it, which is the integral in the logarithm on the right-hand side of (3.28), is expanded in exactly the same way as in Sect. 7 [I]. The differences, like the restriction of the integration to $\Lambda_{k+1}$, or the larger class of localization domains, do not matter, and the factor is represented as $\exp \widetilde{\mathbf{E}}^{(k+1)}\left(\Lambda_{k+1}\right)$. The expression $\widetilde{\mathbf{E}}^{(k+1)}\left(\Lambda_{k+1}\right)$ in the exponential is given by (I.7.12), with terms given by (I.7.13), but with the localization domains $Z_{\mathrm{i}} \in \mathbf{D}_{\mathrm{k}}$, $X \in \mathbf{D}_{k+1}$ having nonempty intersections with $\Lambda_{k+1}$. The integral in the nominator of the expectation value is expanded in the following way. We take the expansion of the integrated function, and we obtain the sum of integrals of the localized terms. In each integral we apply the Mayer expansion (I.7.1). To each term of the obtained sum we apply next the constructions of Sect. 7 [I], described between (7.2) and (7.10) there. We obtain

[the nominator of the expectation value in (3.37)] $=\sum_{\left\{Z_{0}, \ldots, Z_{n}\right\}} H^{\prime}\left(Z_{0}\right) \cdot \ldots \cdot H\left(Z_{n}\right)$,

where the localization domains $Z_{0}, \ldots, Z_{n}$ satisfy all the conditions described in Sect. 7 [I] in connection with the formula (7.11), and the above conditions too. In addition the domain $Z_{0}$ contains the bond $b$ (or the point $z$ ). We can have $n=0$, for $n>0$ the factors $H\left(Z_{i}\right)$ are the same as in the expansion of the denominator. The factor $H^{\prime}\left(Z_{0}\right)$ is different; it is given by an integral with the integrand including, as one of the factors, a localized term from the expansion of the integrated function in the expectation value in (3.37). Although different, it satisfies the same bound as the 
other factors. In the sum (3.44) we separate the summation over $Z_{0}$, and for a fixed $Z_{0}$ we consider the remaining sum over $\left\{Z_{1}, \ldots, Z_{n}\right\}$. It has the form (I.7.11), with the additional condition that the domains $Z_{i}$ do not intersect $Z_{0}$ along a cube, or a wall of a cube. This condition can be formulated as $Z_{i} \subset \bar{Z}_{0}^{c}$, where $\bar{Z}_{0}^{c}$ is the union of all cubes from $\pi_{k}$, such that they do not intersect $Z_{0}$, or intersect it along a twodimensional edge at most. The sum is exponentiated, and the exponent is represented by (I.7.12), with the additional restriction on the polymers $Z_{i}$. We denote the exponent by $\widetilde{\mathbf{E}}^{(k+1)}\left(\Lambda_{k+1}, \bar{Z}_{0}^{c}\right)$, and we have

$$
\begin{aligned}
\text { [the expectation value in }(3.37)]= & \sum_{Z_{0} \supset b} H^{\prime}\left(Z_{0}\right) \exp \left[\widetilde{\mathbf{E}}^{(k+1)}\left(\Lambda_{k+1}, \bar{Z}_{0}^{c}\right)\right. \\
& \left.-\widetilde{\mathbf{E}}^{(k+1)}\left(\Lambda_{k+1}\right)\right] .
\end{aligned}
$$

The difference in the exponential has the representation (I.7.12) with $\left(Z_{1}, \ldots, Z_{n}\right)$ satisfying the additonal condition that at least one of the localization domains intersects $Z_{0}$ along a three-dimensional wall at least. Resumming the terms of this representation according to (I.7.13), we obtain

[the expectation value in (3.37) $]=\sum_{Z_{0} \supset b} H^{\prime}\left(Z_{0}\right) \exp \left[-\sum_{Y} \widetilde{\mathbf{E}}^{(k+1)}\left(\Lambda_{k+1}, Y\right)\right]$.

The sum in the exponential is over the localization domains $Y$ satisfying both conditions relative to $\Lambda_{k+1}, Z_{0}$. Finally, the exponential on the right-hand side of (3.46) is expanded into the Mayer expansion, as the action density (I.7.1). This, after the proper resummation, yields the representation

$$
\text { [the expectation value in (3.37)] }=\sum_{X \in \mathbf{D}_{k+1}, X \supset b} \mathbf{E}_{0}^{(k+1)}\left(\Lambda_{k+1}, X, b\right) .
$$

There is also the dependence on the variable $t$, suppressed in the above formula. The terms of the sum above satisfy the bounds (2.42), with the constant $B_{0}$ replaced by $O\left(p_{0}^{3}\left(g_{k}\right)\right)$. Of course the integration with respect to $t$ preserves the form of the representation, and the multiplication by $g_{k}$ yields small bounds.

The second integral on the right-hand side of (3.37) is treated in the same way as above, but it is simpler because there is only the Gaussian action. Thus we start with the expansion (I.7.3), taken with $Y_{0}=\emptyset$, for the denominator, and with this expansion taken with $Y_{0}=\square, \square$ is the cube containing $b$, for the nominator. Next we repeat the steps from (I.7.3) to (I.7.13), and the above from (3.44) to (3.47). We obtain an expansion of the form (3.47), with terms satisfying the bounds (I.1.18). Finally, the operators in the first integral on the right-hand side of (3.37) are represented by the generalized random walk expansions. After the integration we get a representation of the form (3.47), with terms satisfying (2.42).

Summing up the above expansions we obtain the representation (3.47) for $\mathbf{E}_{0}^{(k+1)}\left(\Lambda_{k+1}, b\right)$, and the corresponding representation for $\mathbf{E}_{0}^{(k+1)}\left(\Lambda_{k+1}, z\right)$. The important remark is that the terms $\mathbf{E}_{0}^{(k+1)}\left(\Lambda_{k+1}, X, z\right)$ of this representation, for $X \subset \Lambda_{k+1}$, do not depend on $\Lambda_{k+1}$, and coincide with the corresponding terms arising from the expressions defined on the whole lattice, i.e. in the framework of [I]. Now we can define the expressions in the formulas (2.26), (2.27) for $j=k+1$. 
We put $\mathbf{E}^{(k+1)}(X, z)=\mathbf{E}_{0}^{(k+1)}\left(\Lambda_{k+1}, X, z\right)$ for $z \in \Lambda_{k+1}^{0}, X \subset \Lambda_{k+1}$, and we define $\mathbf{E}^{(k+1)}\left(\Lambda_{k+1}, z\right), \mathbf{E}^{(k+1)}\left(\Lambda_{k+1}\right)$ by the formulas (2.27), (2.26) correspondingly. This ends the construction of the regular part of the effective action after $k+1$-st renormalization transformation. The remaining terms in the expansions (3.47) are included into the boundary term $\mathbf{B}^{(k+1)}$.

The other expectation values in (3.26), (3.28) are treated in exactly the same way. We have discussed already the localized expansions for the actions defining the corresponding measures, and for the integrated expressions. Having these expansions we proceed with the cluster expansions, and with the operations described above in (3.44)-(3.47). In effect we get representations of the type (3.47). Let us describe briefly special features of these representations for various expectation values. This in (3.28) is almost identical to the one in (3.37), but the expressions integrated have localized expansions with localization domains always intersecting $\Omega_{k+1} \backslash \Lambda_{k+1}$, hence this whole expectation value is included into the boundary term $\mathbf{B}^{(k+1)}$. Similarly, the first expectation value in (3.26) is included into this boundary term, too. The expansion (3.47) for the second expectation value in (3.26) is divided into two parts. The terms with localization domains contained in $\Lambda_{k+1}$ have the same important property as the corresponding $\mathbf{E}^{(k+1)}$-terms, namely they do not depend on $\Lambda_{k+1}$. We denote them by $\mathbf{R}^{\prime \prime(k+1)}$, and we include them into the term $\mathbf{R}^{(k+1)}$. The remaining terms are included again into the boundary term.

Thus we have finished the decomposition of the logarithm of the fluctuation field integral in (3.25) into the sum of the three terms: $\mathbf{E}^{(k+1)}, \mathbf{R}^{\prime \prime(k+1)}$, and $\mathbf{B}^{(k+1)}$. These terms satisfy all the conditions of the inductive assumption. Let us remark that they are not the terms in the $k+1$-st effective action, because we have to perform yet the R-operation, which will change many features of the action. Obviously in general new terms will be included into $\mathbf{R}^{(k+1)}$. To complete the procedure in this step, we have to renormalize the new terms. The vacuum energy renormalization is performed by subtracting the values of $\mathbf{E}^{(k+1)}$ and $\mathbf{R}^{\prime \prime(k+1)}$ at the configuration $U_{k+1}=1$ from the corresponding terms, and adding these values to $E_{0}^{(k)}$. This yields the term $E^{(k)}$, and we define $-E_{k}+E^{(k)}=-E_{k+1}$. The coupling constant renormalization is performed by subtracting $\beta_{k+1}\left(g_{k}\right) A\left(\phi_{k+1}, U_{k+1}\right)$ from $\mathbf{E}^{(k+1)}-\mathbf{E}^{(k+1)}(1)$, and by replacing the function $g_{k}^{-2}(\cdot)$ in the action $A\left(g_{k}^{-2}(\cdot), U_{k+1}\right)$ by $g_{k+1}^{-2}(\cdot)$ defined by the equation (2.24) with $j=k+1$. After this we obtain $T \varrho_{k}$ represented exactly in the form described by the inductive assumption with $k+1$ instead of $k$. This ends the inductive proof of this representation.

The Proof of Theorem 2. Here we want to achieve a more extensive goal than just the proof. We want to describe an approach to renormalization, which is alternative to the one described in Sects. 3-5 [I]. It will clarify also some of the issues barely mentioned only in the above analysis.

The basic expression to analyze, either in the proof of Theorem 2 , or in the analysis of renormalization, is the difference $\mathbf{E}^{(j)}\left(\Lambda_{j}, U_{k}, z\right)-\mathbf{E}^{(j)}\left(\Lambda_{j}, 1, z\right)$. The field $U_{k}$ may depend on the fluctuation field, as in the exponential in (3.15). Let us assume that $z \in \Lambda_{j}^{0} \cap\left(\Omega_{n} \backslash \Omega_{n+1}\right)$, and let us take the cube $\square \in \pi_{n}$ such that $z \in \square$. We represent the function $\mathbf{E}^{(j)}\left(\Lambda_{j}, z\right)$ as the sum (2.27) over the localization domains $X \in \mathbf{D}_{j}$, $X \subset \Lambda_{j}, z \in X$. Consider the two cases (I.3.5): $X \subset \square^{\sim 2}, X \cap\left(\square^{\sim 2}\right)^{c} \neq \emptyset$ (the $\sim$ operation is in the $\mathrm{L}^{-n}$-scale). In the second case the function $\mathbf{E}^{(j)}(X, z)$ is already 
very small by the bound (I.1.18):

$$
\left|\mathbf{E}^{(j)}(X, z)\right| \leqq E_{0} \exp \left(-\kappa\left(L^{j} L^{-n}\right)^{-1}\right) \exp \left(-\frac{1}{2} \kappa d_{j}(X)\right)
$$

In the proof of Theorem 2 we simply estimate all these terms using the above bound, and the sum over $X$ is bounded by $O(1) \exp \left(-\kappa\left(L^{j} L^{-n}\right)^{-1}\right) \leqq O(1)\left(L^{j} L^{-n}\right)^{5}$. This is an admissible error contributing only to the constant on the right-hand side of (2.43). For the complete analysis we apply the formulas (3.3), (3.6)-(3.13) [I]. We may use also the simpler representation $\mathbf{E}^{(j)}\left(X, \exp i \eta \mathbf{H} U_{k+1}, z\right)$, and then to replace the background configuration by the corresponding complex variables.

Consider the basic first case now. We keep the same notations as in Sect. 3 [I], and we apply the formulas and transformations (3.3), (3.18)-(3.28) [I]. They have a bit different meaning now, because the configuration $U_{k}$, or $U_{k+1}$, restricted to $\Omega_{n} \backslash \Omega_{n+1}$ coincides with a $U_{n}$-type confiuration, but the difference is seen in bounds only. Notice also that for the proof of Theorem 2 we put $\mathbf{H}_{k}\left(B^{\prime}\right)=0$ in (I.3.28), or $\mathbf{A}=0$ in (I.3.30), and we replace $\mathrm{L}^{-1} \eta \mathbf{H}_{k+1}$ by $\eta \mathbf{H}_{k}$. The function $\mathrm{L}^{n} \eta A$, where $A$ is defined by (I.3.30), satisfies the bound (I.3.32), but in the $\mathrm{L}^{-n}$-scale instead of the $\eta$-scale. Next we apply the expansion (I.3.34). The last term in it satisfies the bound (I.3.54), the right-hand side of which can be further bounded by (I.3.35), again with $\eta$ replaced by $L^{-n}$. To the sum on the right-hand side (I.3.34) we apply the considerations of Sect. 4 [I], with two differences only. We do not differentiate with respect to $t_{\square}$ yet, hence all the factors $B$ in the sum are the same, and applying the Ward-Takahashi identities (I.4.14), (I.4.15), and other operations of that section, we move the factors $B$ to the point $z$ instead of the point $x$. The final formula we obtain is slightly different from the formula (I.4.34). We have

$$
\begin{aligned}
\sum_{n=1}^{4} \frac{1}{n !}\left\langle\mathbf{E}^{(n)}(X, z), \stackrel{\otimes}{\otimes} B\right\rangle= & \sum_{\mu, v, \kappa, \lambda}\left[\sum_{x, y} \mathbf{E}_{\mu \nu}^{(2)}(X, x, y, z)\left(x_{\kappa}-z_{\kappa}\right)\left(y_{\lambda}-z_{\lambda}\right)\right] \\
& \cdot \frac{1}{2} \operatorname{tr}\left(\left(\partial_{\kappa} B_{\mu}\right)(z)+\frac{1}{2} i\left[B_{\kappa}(z), B_{\mu}(z)\right]\right) \\
& \cdot\left(\left(\partial_{\lambda} B_{v}\right)(z)+\frac{1}{2} i\left[B_{\lambda}(z), B_{v}(z)\right]\right) \\
& +(\text { the irrelevant terms })
\end{aligned}
$$

As in Sect. 4 [I] we have dropped the superscript $(j)$ in the symbols above, the superscripts written denote the functional derivatives with respect to $B$. In particular

$$
\mathbf{E}_{\mu \nu}^{(2)}(X, x, y, z)=\left.\frac{\delta^{2}}{\delta B_{\mu}(x) \delta B_{v}(y)} \mathbf{E}^{(j)}\left(X, U_{j}(\exp i B), z\right)\right|_{B=0} .
$$

This implies $\mathbf{E}_{\mu \nu}^{(2)}(X, x, y, z)=\mathbf{E}_{v \mu}^{(2)}(X, y, x, z)$. We prove the following antisymmetry property

$$
\sum_{x} \mathbf{E}_{\mu \nu}^{(2)}(X, x, y, z)\left(x_{\kappa}-z_{\kappa}\right)=-\sum_{x} \mathbf{E}_{\kappa v}^{(2)}(X, x, y, z)\left(x_{\mu}-z_{\mu}\right)
$$


Let us suppress the other symbols and denote the left-hand side above by $\mathbf{E}_{\mu \kappa}$. We decompose it into the symmetric and antisymmetric parts:

$$
\mathbf{E}_{\mu \kappa}=\mathbf{E}_{\mu \kappa}^{(s)}+\mathbf{E}_{\mu \kappa}^{(a)}, \quad \mathbf{E}_{\mu \kappa}^{(s)}=1 / 2\left(\mathbf{E}_{\mu \kappa}+\mathbf{E}_{\kappa \mu}\right) .
$$

We have to prove that the symmetric part is equal to 0 . Take the scalar product with an arbitrary matrix $A, \operatorname{tr} A \mathbf{E}^{(s)}=\operatorname{tr} A^{(s)} \mathbf{E}$, where $A^{(s)}$ is the symmetric part of $A$. Thus

$$
\operatorname{tr} A \mathbf{E}^{(s)}=\sum_{x, \mu} \mathbf{E}_{\mu \nu}^{(2)}(X, x, y, z) \sum_{\kappa} A_{\mu \kappa}^{(s)}\left(x_{\kappa}-z_{\kappa}\right) .
$$

Take the function

$$
\lambda(x)=\frac{1}{2} \sum_{\mu, \kappa} A_{\mu \kappa}^{(s)}\left(x_{\mu}-z_{\mu}\right)\left(x_{\kappa}-z_{\kappa}\right)-\sum_{\mu} A_{\mu \mu}^{(s)}\left(x_{\mu}-z_{\mu}\right) .
$$

Of course $\left(\partial_{\mu} \lambda\right)(x)=\sum_{\kappa} A_{\mu \kappa}^{(s)}\left(x_{\kappa}-z_{\kappa}\right)$, hence

$$
\operatorname{tr} A \mathbf{E}^{(s)}=\sum_{x, \mu}\left(\partial_{\mu} \lambda\right)(x) \mathbf{E}_{\mu \nu}^{(2)}(X . x, y, z)=0
$$

by the first identity (I.4.15). Because $A$ is an arbitrary matrix, so the above equalities imply $\mathbf{E}^{(s)}=0$, which is the property (3.51). Denoting by $\mathbf{E}_{\mu \nu, \kappa \lambda}(X, z)$ the sum over $x, y$ in the square bracket in (3.49), we have

$$
\mathbf{E}_{\mu \nu, \kappa \lambda}^{(2)}(X, z)=-\mathbf{E}_{\kappa v, \mu \lambda}^{(2)}(X, z)=-\mathbf{E}_{\mu \lambda, \kappa v}^{(2)}(X, z) .
$$

This property allows us to antisymmetrize the derivatives in (3.49) in the indices $\mu, \kappa$ and $v, \lambda$. Denoting $F_{\kappa \mu}(z)=\left(\partial_{\kappa} B_{\mu}\right)(z)-\left(\partial_{\mu} B_{\kappa}\right)(z)+i\left[B_{\kappa}(z), B_{\mu}(z)\right]$, we have

$$
\begin{aligned}
\sum_{n=1}^{4} \frac{1}{n !}\left\langle\mathbf{E}^{(n)}(X, z), \stackrel{\otimes}{\otimes} B\right\rangle= & \sum_{\kappa<\mu, \lambda<v} \frac{1}{2} \mathbf{E}_{\mu \nu, \kappa \lambda}^{(2)}(X, z) \operatorname{tr} F_{\kappa \mu}(z) F_{\lambda v}(z) \\
& +(\text { the irrelevant terms) } .
\end{aligned}
$$

Let us recall that (the irrelevant terms) above, and in (3.49), denotes the sum of terms which can be bounded by $O\left(\left(L^{j} L^{-n}\right)^{5-\beta}\right) \exp \left(-\kappa d_{j}(X)\right)$, where $\beta$ is a positive number. The identities (3.56) hold for the localization domains $X$ satisfying the condition $X \subset \square^{\sim 2}$. We sum up the identities over all such domains, and we extend the sum on the right-hand side to all domains $X \in \mathbf{D}_{j}$ for the space $L^{-j} Z^{d}, X$ containing the point $z$. The difference between the two sums contributes to the irrelevant terms only, by the bounds (3.48). Thus we obtain the identity (3.56) resummed over the domains $X$, but with the first expression on the right-hand side replaced by

$$
\frac{1}{2} \sum_{\kappa<\mu, \lambda<v} \Pi_{\mu \nu, \kappa \lambda}^{(j)} \operatorname{tr} F_{\kappa \mu}(z) F_{\lambda v}(z) .
$$

Here $\Pi_{\mu \nu, \kappa \lambda}^{(j)}=\sum_{\lambda, y} \Pi_{\mu \nu}^{(j)}(x, y, z)\left(x_{\kappa}-z_{\kappa}\right)\left(y_{\lambda}-z_{\lambda}\right)$, and the function $\Pi_{\mu \nu}^{(j)}(x, y, z)$ is given by the formula (3.50), but with $\mathbf{E}^{(j)}\left(X . U_{j}, z\right)$ replaced by $\mathbf{E}^{(j)}\left(U_{j}, z\right)$ defined on the whole lattice $L^{-j} Z^{d}$. This function is translation invariant, hence $\Pi_{\mu \nu, \kappa \lambda}^{(j)}$ is independent of $z$. Let us describe its other properties. The most important one is the 
Euclidean covariance following from (2.29). It can be reformulated as

$$
\mathbf{E}^{(j)}\left(U_{j}(\exp i r B), r z\right)=\mathbf{E}^{(j)}\left(U_{j}(\exp i B), z\right),
$$

where $r B$ represents the configuration $(r B)_{\mu}(x)=(r B)_{\mu}\left(r^{-1} x\right)$. The notation here is confusing, the symbol $r$ on the left-hand side denotes the transformation of the vector field on the lattice $Z^{d}$, generated by the Euclidean transformation $r$ of this lattice. On the right-hand side $r$ denotes the matrix of the corresponding rotation, and $r^{-1} x$ denotes the point obtained by the application of $r^{-1}$ to $x$. We must also take into account the convention $B\left(\left\langle x, x+e_{\mu}\right\rangle\right)=B_{\mu}(x)$. For example, if $r$ is the reflection in the hyperplane $x_{\mu}=0$, then

$$
\begin{aligned}
(r B)_{\mu}(x) & =(r B)\left(\left\langle x, x+e_{\mu}\right\rangle\right)=B\left(r\left\langle x, x+e_{\mu}\right\rangle\right)=-B\left(r\left\langle x+e_{\mu}, x\right\rangle\right) \\
& =-B\left(\left\langle\left(\ldots,-x_{\mu}-1, \ldots\right),\left(\ldots,-x_{\mu}, \ldots\right)\right\rangle\right)=-B_{\mu}\left(\left(\ldots,-x_{\mu}-1, \ldots\right)\right) .
\end{aligned}
$$

Thus $r x=\left(\ldots,-x_{\mu}, \ldots\right)-e_{\mu}$, which is equal to $r x-e_{\mu}$, where in the last expression $r x$ denotes simply the reflection applied to the point $x$. Keeping in mind these different meanings of the symbol $r$, we write the following conclusion of the covariance (3.58)

$$
\left(\left(r^{-1} \otimes r^{-1}\right) \Pi^{(j)}\right)_{\mu \nu}(r x, r y, r z)=\Pi_{\mu \nu}^{(j)}(x, y, z) .
$$

For the number $\Pi_{\mu \nu, \kappa \lambda}^{(j)}$ these different interpretations of $r$ disappear, and the above covariance together with the first Ward-Takahashi indentity (I.4.15) imply

$$
\left((\stackrel{4}{\otimes} r) \Pi^{(j)}\right)_{\mu \nu, \kappa \lambda}=\Pi_{\mu \nu, \kappa \lambda}^{(j)} .
$$

Here $r$ denotes the matrix of the Euclidean rotation $r$. This invariance has the usual implications, analyzed already in Sect. 5 [I]. Let us describe them for the indices satisfying the restrictions $\kappa<\mu, \lambda<v$ occurring in the sum (3.57). Considering reflections we conclude that in this case the coefficients in the sum are different from 0 only if $\mu=v$ and $\kappa=\lambda$. Considering permutations we conclude that then they are all equal, e.g. they are equal to their values for $\mu=v=2, \kappa=\lambda=1$. Thus we obtain

$$
(3.57)=\beta_{j}^{\prime} \frac{1}{2} \sum_{\mu<v} \operatorname{tr} F_{\mu v}^{2}(z), \quad \beta_{j}^{\prime}=\Pi_{22,11}^{(j)} .
$$

The last identity can be considered as a possible definition of the $\beta$-function. Now we prove that it coincides with the definition (I.1.22). Using the translation invariance and the identity (I.4.15) again we have

$$
\begin{aligned}
\beta_{j}^{\prime} & =\sum_{x, y} \Pi_{22}^{(j)}(x, y, 0) x_{1} y_{1}=-\frac{1}{2} \sum_{x, y} \Pi_{22}^{(j)}(x, y, 0)\left(x_{1}-y_{1}\right)^{2} \\
& =-\frac{1}{2} \sum_{x, y} \Pi_{22}^{(j)}(z-y, 0,-y)\left(x_{1}-y_{1}\right)^{2}=-\frac{1}{2} \sum_{x, z} \Pi_{22}^{(j)}(x, 0, z) x_{1}^{2} .
\end{aligned}
$$

From the equality (2.26) for $\Lambda_{j}=L^{-j} Z^{d}$, and from the definitions (I.1.20), (I.5.1), we obtain

$$
\sum_{z} \Pi_{\mu \nu}^{(j)}(x, y, z)=\Pi_{\mu \nu}^{(j)}(x-y)
$$


Thus the representation (I.5.16), or (I.5.37), implies

$$
\beta_{j}^{\prime}=-\frac{1}{2} \sum_{z} \Pi_{22}^{(j)}(x) x_{1}^{2}=\frac{1}{2}\left(\frac{\partial^{2}}{\partial p_{1}^{2}} \Pi_{22}^{(j)}\right)(0)=\beta_{j} .
$$

This is the required equality.

Consider now the term $-\beta_{j} A\left(\phi_{j}, U_{k}\right)$. We decompose it into the sum of localized expressions

$$
A\left(\phi_{j}, U_{k}\right)=\sum_{z \in T^{(j)}} A\left(h_{z} \phi_{j}, U_{k}\right)
$$

where $h_{z}$ is defined as in (3.40), but on the lattice $T_{L^{-\jmath}}$, and with $h(t)=\max \{1-|t|, 0\}$. For $z \in \Lambda_{j}^{0}$ we have $h_{z} \phi_{j}=h_{z}$, and the function $A\left(h_{z}, U_{j}\right)$ is Euclidean covariant. Analyzing $A\left(h_{z}, U_{k}\right)$ in the same way as above, we obtain

$$
A\left(h_{z}, U_{k}\right)=\frac{1}{2} \sum_{\mu<v} \operatorname{tr} F_{\mu v}^{2}(z)+(\text { the irrelevant terms }) .
$$

Thus the first expression on the right-hand side of the expansion of $-\beta_{j} A\left(h_{z}, U_{k}\right)$ cancels the expression on the right-hand side of (3.61), and we are left with the irrelevant terms only. To get the expressions needed in the fluctuation field integral, we differentiate these terms with respect to the parameter $t_{\square}$ multiplying the fluctuation field. For the expressions considered in the proof of Theorem 2, we have the following conclusion:

$$
\mathbf{E}^{(j)}\left(\Lambda_{j}, U_{k}, z\right)-\mathbf{E}^{(j)}\left(\Lambda_{j}, 1, z\right)-\beta_{j} A\left(h_{z}, U_{k}\right)=O\left(\left(L^{j} L^{-n}\right)^{5-\beta}\right),
$$

for $z \in \Lambda_{j}^{0} \cap\left(\Omega_{n} \backslash \Omega_{n+1}\right), \beta>0$. Summing over $z \in \Lambda_{j}^{0} \cap \Omega \mathrm{w}$ get the inequality (2.43) in Theorem 2 (with $1-\beta, \beta>0$, instead of $\beta<1$ ). Thus we have proved the first part of Theorem 2, concerning the functions $\mathbf{E}^{(j)}$.

The inequality (2.44) for the functions $\mathbf{R}^{(j)}$ can be proved in an almost identical way. We analyze these functions as above, but we stop at the identity (3.49), where $\mathbf{E}^{(2)}(X, x, y, z)$ is replaced by $\mathbf{R}^{(2)}(X, x, y)$, and $z$ is an arbitrary point from $X$. Now all the terms on the right-hand side can be bounded by $O(1)\left(L^{j} L^{-n}\right)^{4} g_{j}^{\kappa_{0}} \exp \left(-\kappa d_{j}(X)\right)$, and this yields the inequality (2.44). The proof of Theorem 2 is completed.

Finally, let us sketch briefly another proof of Theorem 2. It is interesting because we do not use the Ward-Takahashi identities, but directly the gauge invariance. As in the previous proof we want to analyze the difference $\mathbf{E}^{(j)}\left(\Lambda_{j}, U_{k}, z\right)-\mathbf{E}^{(j)}\left(\Lambda_{j}, 1, z\right)$ for $z \in \Lambda_{j}^{0} \cap\left(\Omega_{n} \backslash \Omega_{n+1}\right)$. Now we take the cube $\square \in \pi_{j}$ containing $z, z \in \square$, and the cube $\square^{\sim n-j}$. We divide the localization domains $X$ into two classes: $X \subset \square^{\sim n-j}$, or $X \cap\left(\square^{\sim n-j}\right)^{c} \neq \emptyset$. For $X$ in the second class the difference of the corresponding expressions can be estimated by $2 E_{0} \exp (-(1 / 2) \kappa(n-j)) \exp \left(-(1 / 2) \kappa d_{j}(X)\right)$, and $\exp (-(1 / 2) \kappa(n-j))<\left(L^{j} L^{-n}\right)^{5}$, hence these terms can be treated as before. Consider the terms with localization domains in the first class. Take the cube $\square_{0}=\square^{\sim n-j+1}$ and the representation $U_{k}$ $=U_{j, \square_{0}}\left(M^{*}\left(U_{k}\right)\right)$. Now we repeat the construction of Sect. F [15], and we introduce the axial gauge for the field $M^{j}\left(U_{k}\right)$ on $\square_{0}$, considering $\square_{0}$ as one block with center at $z$. We denote the argument in the representation by $V^{\prime}$, i.e., $V^{\prime}$ 
$=M^{\circ}\left(U_{k}\right)$, and $V^{\prime}$ is a small field, more precisely we have

$$
\begin{aligned}
\left|V^{\prime}-1\right| & <11 d^{2} \varepsilon_{n}\left(L^{j} L^{-n}\right)^{2}+d M(n-j+2) \varepsilon_{n}\left(L^{j} L^{-n}\right)^{2} \\
& <3 d M\left(1+\log L^{j} L^{-n}\right) \varepsilon_{n}\left(L^{j} L^{-n}\right)^{2} .
\end{aligned}
$$

We write $V^{\prime}=\exp i B^{\prime}$, and we expand the terms up to the third order in $B^{\prime}$. The third order terms are already irrelevant by the above bound, hence

$$
\begin{aligned}
\mathbf{E}^{(j)}\left(X, U_{k}, z\right)-\mathbf{E}^{(j)}(X, 1, z)= & \frac{1}{2}\left\langle\frac{\delta^{2}}{\delta B^{\prime 2}} \mathbf{E}^{(j)}\left(X, U_{j, \square 0}(1), z\right), B^{\prime}, B^{\prime}\right\rangle \\
& +O(1)\left(L^{j} L^{-n}\right)^{6-\beta} \exp \left(-\kappa d_{j}(X)\right) .
\end{aligned}
$$

In the quadratic form above we restrict $B^{\prime}$ to $\square^{\sim n-j}$, and we replace the function $U_{j, \square_{0}}$ by $U_{j}$, the differences contribute to the last term only. Summing over $X$ we get

$$
\begin{aligned}
\mathbf{E}^{(j)}\left(\Lambda_{j}, U_{k}, z\right)-\mathbf{E}^{(j)}\left(\Lambda_{j}, 1, z\right)= & \frac{1}{2} \sum_{x, y, \mu, v} \Pi_{\mu \nu}^{(j)}(x, y, z) \operatorname{tr} B_{\mu}^{\prime}(x) B_{v}^{\prime}(y) \\
& +O(1)\left(L^{j} L^{-n}\right)^{5},
\end{aligned}
$$

where the summation over $x, y$ is restricted to $\square^{\sim n-j}$, and

$$
B^{\prime}(b)=\frac{1}{i} \log \bar{U}_{k}^{j}\left(\Gamma_{z, b_{-}} \cup b \cup \Gamma_{b_{+}, z}\right)
$$

Now we take the cube $\square_{1} \in \pi_{n}$ containing $z$, and we repeat the construction before (3.68), but for the representation $U_{k}=U_{n, \square}\left(M^{*}\left(U_{k}\right)\right)$. We write $U_{k}$ $\left.=\left(\exp i L^{-n} \mathbf{H}_{n, \square \tilde{1}}(1 / i) \log M^{\cdot}\left(U_{k}\right)\right)\right)^{u_{k}^{-1}}$, and using the gauge invariance of the trace in (3.70) we can replace the variables $B^{\prime}$ by

$$
\begin{aligned}
\frac{1}{i} \log \left(\exp i Q_{j}\left(L^{-n} \mathbf{H}_{n, \square}\right)\right)(\Gamma)= & \frac{1}{i} \log (\exp i B)(\Gamma) \\
= & B(\Gamma)+\frac{1}{2} \sum_{b, b^{\prime} \subset \Gamma, b<b^{\prime}} i\left[B(b), B\left(b^{\prime}\right)\right] \\
& +O(1)\left(L^{j} L^{-n}\right)^{3}
\end{aligned}
$$

where $\Gamma=\Gamma_{z, b_{-}} \cup b \cup \Gamma_{b_{+}, z}$. The contour variable $B(\Gamma)$ is represented as $(\partial B)(\Sigma)$, where $\Sigma$ is a minimal surface with the boundary $\Gamma$. This assures that both terms on the right-hand side above are of the order $O\left(\left(L^{j} L^{-n}\right)^{2}\right)$. We move the plaquette variables in the first term, and the bond variables in the second term, to the point $z$. The main term equals $\sum_{\kappa<\mu}\left(x_{\kappa}-z_{\kappa}\right) F_{\kappa \mu}(z)$, if $b=\left\langle x, x+e_{\mu}\right\rangle$, the remainder can be estimated by $O(1)\left(L^{j} L^{-n}\right)^{3}$. Thus we obtain the representation (3.70), but with the first term on the right-hand side replaced by the expression (3.57). The remaining arguments are as in the first proof.

The above proof can be also elaborated to a complete renormalization procedure, as the one in Sects. 3-5 of [I], but it is important to notice that it works only if we have the representation (2.26), (2.27) with the corresponding properties, similarly as the first proof. 
Acknowledgements. The author would like to express his gratitude for numerous interesting discussions during his work on gauge field theories to his collaborators Profs. A. Jaffe, J. Imbrie, D. Brydges, and to Profs. G. Gallavotti, F. Nicolo, G. Benfatto, R. Sénéor, J. Magnen, V. Rivasseau, and J. Feldman.

\section{References}

I Bałaban, T.: Renormalization group approach to lattice gauge field theories. I. Generation of cffective actions in a small field approximation and a coupling constant renormalization in four dimensions. Commun. Math. Phys. 109, 249-301 (1987) and references therein

II Bałaban, T.: Renormalization group approach to lattice gauge field theories. II. Cluster expansions. Commun. Math. Phys. 116, 1-22 (1988)

Communicated by A. Jaffe

Received June 5, 1987, in revised form February 8, 1988 
